\title{
TITLE:
}

\section{Cohen-Macaulay approximations from the viewpoint of triangulated categories}

$\operatorname{AUTHOR}(\mathrm{S}):$

Kato, Kiriko

\section{CITATION:}

Kato, Kiriko. Cohen-Macaulay approximations from the viewpoint of triangulated categories. 数理解析研究所講究録 1996, 964: 1-28

ISSUE DATE:

1996-08

URL:

http://hdl.handle.net/2433/60581

RIGHT: 


\title{
Cohen-Macaulay approximations from the viewpoint of triangulated categories
}

\author{
Kiriko Kato \\ Department of Mathematics \\ Ritsumeikan University, Kusatsu, 525-77, Japan \\ Tel: +81-775-66-1111 Fax: +81-775-61-2657 \\ e-mail: kiriko@kurims.kyoto-u.ac.jp
}

\section{Introduction}

Let $(R, \mathfrak{m}, k)$ be a complete Gorenstein local ring, and let $M$ be a finitely generated $R$-module. Auslander and Buchweitz introduced the notion of CohenMacaulay approximation (1.1) and a finite projective hull (1.2), of $M$, which are the exact sequences dual to each other [1], [5] :

$$
\begin{gathered}
0 \rightarrow Y_{M}^{R} \rightarrow X_{M}^{R} \stackrel{\rho_{M}}{\rightarrow} M \rightarrow 0, \\
0 \rightarrow M \stackrel{\zeta^{M}}{\rightarrow} Y_{R}^{M} \stackrel{u_{M}}{\rightarrow} X_{R}^{M} \rightarrow 0,
\end{gathered}
$$

where $X_{M}^{R}, X_{R}^{M}$ are maximal Cohen-Macaulay modules and $Y_{M}^{R}, Y_{R}^{M}$ are $\bmod$ ules of finite projective dimension.

If $X_{M}^{R}$ and $Y_{M}^{R}$ (resp. $X_{R}^{M}$ and $Y_{R}^{M}$ ) have no direct summand in common, according to the inclusion map appeared in the sequence (1.1) (resp. the projection map in the sequence (1.2)), it is called the minimal Cohen-Macaulay approximation (resp. the minimal finite projective hull), which exists uniquely up to isomorphisms. We may assume henceforth the minimality of (1.1) and (1.2), omitting common summands if necessary.

The above exact sequences suggest an idea to treat a finite module as a kernel or a cokernel of a homomorphism from a finite projective dimensional module to a Cohen-Macaulay module. Indeed, on researching Cohen-Macaulay approximations, there arises a natural question: If $X_{M} \cong X_{N}, Y_{M} \cong Y_{N}$, do two modules $M$ and $N$ share any common property? We discuss the problem within a framework of the theory of triangulated categories; which in this case consists of Cohen-Macaulay modules, finite projective modules, and finitely generated modules over $R$. In addition to above two exact sequences (Cohen-Macaulay approximation and finite projective hull), in the section2 we 
construct another exact sequence "original extension" which is the dual of the other two. For original extensions, as well as Cohen-Macaulay approximations and finite projective hulls, we define the minimality, though it is not that simple. The notion of original extensions enables us to consider two $R$-modules $M$ and $N$ with $X_{M} \cong X_{N}, Y^{M} \cong Y^{N}$ as two elements of an $R$-module $\operatorname{Ext}_{R}^{1}\left(Y^{M}, \Omega_{R}^{1}\left(X^{M}\right)\right)$.

Unlike a Cohen-Macaulay approximation and a finite projective hull, a non-minimal original extension does not always includes the minimal original extension. The existence of a non-trivial non-minimal original extension obstructs the uniqueness of the correspondence between finite modules and elements of the module of the form $\operatorname{Ext}_{R}^{1}(Y, X)$. Even though, our Lemma 2.5 shows that $\operatorname{Ext}_{R}^{1}\left(Y^{M}, \Omega_{R}^{1}\left(X^{M}\right)\right.$ ) (where the minimal original extension of $M$ sits) contains a non-trivial non-minimal original extension if and only if $M$ is reducible; $M=$ Coker $g f$ for some linear maps $f, g$ between free modules with $\Omega_{R}^{1}(M)=\Omega_{R}^{1}($ Coker $f)$. In other words, due to that complexity, we can investigate the homological structure of a module $M$ via $\operatorname{Ext}_{R}^{1}\left(Y^{M}, \Omega_{R}^{1}\left(X^{M}\right)\right)$.

The ensuing section3 deals with chasing the Cohen-Macaulay approximations ( finite projective hulls or original extensions) through $R$ homomorphisms. For a homomorphism $f: M \rightarrow N$ of modules, we construct Cohen-Macaulay approximations, etc. of Coker $f$ or $\operatorname{Ker} f$, from those of $M$ and $N$. While we extended Auslander's delta-invariants, defined with respect to Cohen-Macaulay approximations, to three types of invariants, each of which belongs to three exact sequences Cohen-Macaulay approximations, finite projective hulls, and original extensions. And moreover, we observed the change of these invariants according to homomorphisms. Those method are applicable to concern with the lifting problem; namely how the Cohen-Macaulay approximations are inherited through ring homomorphisms.

First of all, let us set the notations used throughout the paper. Over the Gorenstein local ring $(R, \mathfrak{m}, k)$, a "module" always means a finitely generated module. An $R$-complex $F_{\bullet}=\left(F_{\bullet}, d_{F}\right)$ denotes a complex of $R$-modules:

$$
\cdots \rightarrow F_{n} \stackrel{d_{F n}}{\rightarrow} F_{n-1} \rightarrow \cdots
$$

The $n$-th truncated complex $\tau_{n} F$ is defined as

$$
\left(\tau_{n} F\right)_{m}=\left\{\begin{array}{ll}
F_{m} & (m \geq n) \\
0 & (m<n)
\end{array}, \quad d_{\tau_{n} F_{m}}= \begin{cases}d_{F_{m}} & (m>n) \\
0 & (m \leq n)\end{cases}\right.
$$

The shifting complex $F_{\bullet}(n)$ is as follows:

$$
\left(F_{\bullet}(n)\right)_{m}=F_{n+m}, \quad d_{F_{\bullet}(n)_{m}}=d_{F n+m} .
$$

We use the notation $M \stackrel{s t}{\cong} N$ which means that two modules $M$ and $N$ are isomorphic up to free summands. 


\section{Original Extensions}

Definition 2.1 For a finite $R$-module $M$, an original extension of $M$ is the exact sequence

$$
0 \rightarrow X \stackrel{\xi}{\rightarrow} M \oplus P \stackrel{\zeta}{\rightarrow} Y \rightarrow 0
$$

with a Cohen-Macaulay module $X$, a free module $P$, and a finite projective dimensional module $Y$.

An original extension (2.3) is called minimal if it satisfies the following conditions:

1) A Cohen-Macaulay module $X$ is stable.

2) There exists no common summand with $P$ and $Y$ through $\zeta$.

3) For any original extension $0 \rightarrow X^{\prime} \rightarrow M \oplus P^{\prime} \rightarrow Y^{\prime} \rightarrow 0$ of $M$, linear maps $a: P \rightarrow P^{\prime}, b: Y \rightarrow Y^{\prime}$, and $c: X \rightarrow X^{\prime}$ exist and make the following diagram commutative.

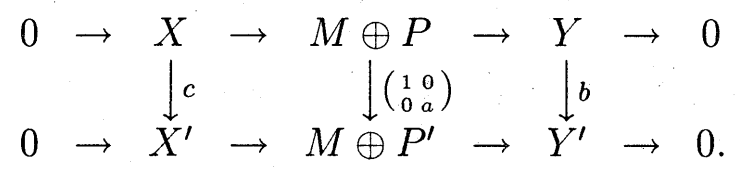

Theorem 2.2 For an $R$-module $M$, there exists a minimal original extension of $M$.

proof) As in the section 3, for the minimal projective hull (1.2) of $M$, take a chain map $u_{M_{\bullet}}: I_{M \bullet} \rightarrow G_{M \bullet}$ such that $\mathrm{H}_{-1}\left(u_{M_{\bullet}}\right)=u_{M}$ for the minimal free resolutions $I_{M \bullet}(-1)$ of $Y^{M}$ and $G_{M \bullet}(-1)$ of $X^{M}$.

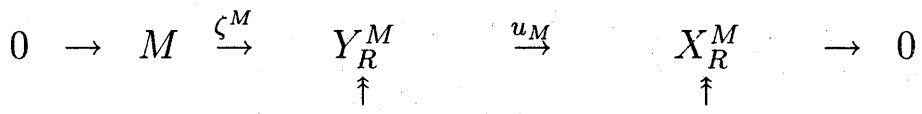

$$
\begin{aligned}
& I_{M \bullet}(-1) \stackrel{u_{M} \cdot(-1)}{\rightarrow} G_{M \bullet}(-1)
\end{aligned}
$$

The exact sequence of the complexes

$$
0 \rightarrow G_{M \bullet} \rightarrow \text { Cone }\left(u_{M \bullet}\right) .(-1) \rightarrow I_{M \bullet}(-1) \rightarrow 0
$$

induces the exact sequence

$$
0 \rightarrow \Omega_{1}^{R}\left(X^{M}\right) \rightarrow \text { Coker } d_{u_{M} 0} \rightarrow Y^{M} \rightarrow 0 .
$$

And we have Coker $d_{u_{M} 0} \cong M \oplus G_{M-1}$ from the split exact sequence

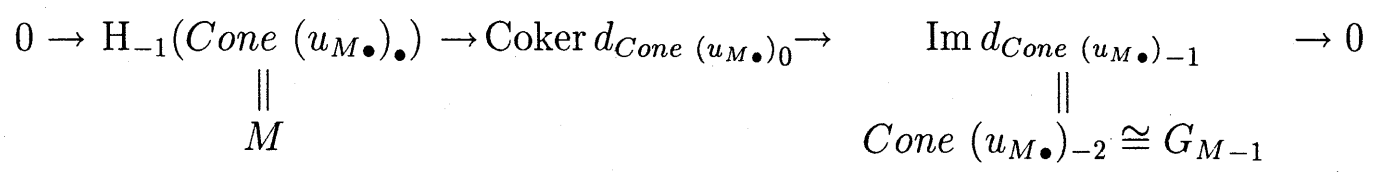


since

$$
\mathrm{H}_{i}\left(\text { Cone }\left(u_{M_{\bullet}}\right)_{\bullet}\right) \cong \begin{cases}\operatorname{Ker} u_{M} \cong M & (i=-1) \\ \operatorname{Coker} u_{M}=0 & (i=-2) \\ 0 & (\text { otherwise })\end{cases}
$$

Consequently, we obtain an original extension of $M$

$$
0 \rightarrow \Omega_{1}^{R}\left(X^{M}\right) \stackrel{\xi}{\rightarrow} M \oplus G_{M-1} \stackrel{\zeta}{\rightarrow} Y^{M} \rightarrow 0 .
$$

After omitting a common free summand of $G_{M}$ and $Y^{M}$ from (2.6), we have an original extension (2.7) of $M$ satisfying the conditions (1) and (2) of the above definition.

$$
0 \rightarrow \Omega_{1}^{R}\left(X^{M}\right) \stackrel{\xi}{\rightarrow} M \oplus P \stackrel{\zeta}{\rightarrow} Z^{M} \rightarrow 0 .
$$

It remains to check the property 3 ) to see the minimality of (2.7). Suppose there exists another original extension of $M$

$$
0 \rightarrow X^{\prime} \stackrel{\xi^{\prime}}{\rightarrow} M \oplus P^{\prime} \stackrel{\zeta^{\prime}}{\rightarrow} Y^{\prime} \rightarrow 0
$$

We shall show the existence of maps that make the diagram (2.4) commutative. On the proof, we may assume $X^{\prime}$ is stable. It follows from the following commutative diagram

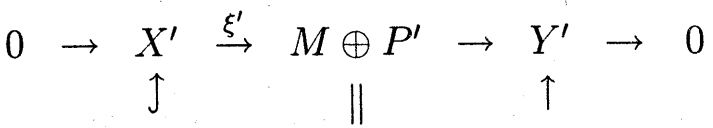

$$
\begin{aligned}
& 0 \rightarrow C^{\prime} \stackrel{\xi^{\prime \prime}}{\rightarrow} M \oplus P^{\prime} \rightarrow Z^{\prime} \rightarrow 0,
\end{aligned}
$$

where $X^{\prime}=C^{\prime} \oplus V$ with a stable Cohen-Macaulay module $C^{\prime}$ and a free module $V$, and $Z^{\prime}$ is of finite projective dimension because of the induced exact sequence $0 \rightarrow V \rightarrow Z^{\prime} \rightarrow Y^{\prime} \rightarrow 0$.

Let $G_{\bullet}(-1)$ be the minimal free resolution of $\Omega_{R}^{-1}\left(X^{\prime}\right)$.

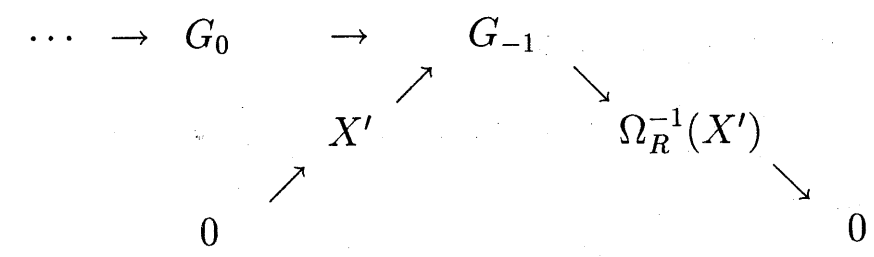

Put $\widetilde{F}_{\bullet}:=F_{M} \oplus P^{\prime}$. where $F_{M \bullet}$ is the minimal free resolution of $M$ and $T_{P^{\prime}} \bullet$ is a trivial complex :

$$
T_{P^{\prime} \bullet}: \underset{(0-t h)}{P^{\prime}}=\underset{(-1-s t)}{P^{\prime}}
$$


We can take a chain map $\tilde{w}_{\bullet}: G_{\bullet} \rightarrow \tilde{F}_{\bullet}$ such that $\mathrm{H}_{0}\left(\tau_{0} \tilde{w}_{\bullet}\right)=\xi$ by the following method. We obtain the map as $\tilde{w}_{\bullet}=x_{M \bullet} \oplus x_{P^{\prime} \bullet}$. First $x_{M_{\bullet}}: G_{\bullet} \rightarrow$ $F_{M}$ is naturally induced by the composite map $\xi_{M}: X^{\prime} \stackrel{\xi}{\rightarrow} M \oplus P^{\prime} \rightarrow M$; $x_{M-1}=0$ and $\mathrm{H}_{0}\left(\tau_{0} x_{M \bullet \bullet}\right)=\xi_{M}$. On the other hand, we define a chain map $x_{P_{\bullet}}: G_{\bullet} \rightarrow P^{\prime}$. as $x_{P 0}:=\xi_{P} d_{G 0}, x_{P-1}:=\operatorname{Hom}_{R}(z, R)$ and $x_{P^{\prime} i}:=0$ to have $\mathrm{H}_{0}\left(\tau_{0} x_{P^{\prime} \bullet \bullet}\right)=\xi_{P^{\prime}}$ where $\xi_{P}$ is the composite $X^{\prime} \stackrel{\xi}{\rightarrow} M \oplus P^{\prime} \rightarrow P^{\prime}$ and $z$ is the map that makes the following diagram commutative:

$$
\begin{array}{ccc}
\operatorname{Hom}_{R}\left(G_{-1}, R\right) & \stackrel{z}{\operatorname{Hom}_{R}\left(P^{\prime}, R\right)} \\
\operatorname{Hom}_{R}\left(X^{\prime}, R\right) & \operatorname{Hom}_{R}\left(\xi_{P^{\prime}}, R\right) & \| \\
\operatorname{Hom}_{R}\left(P^{\prime}, R\right) .
\end{array}
$$

The exact sequence of complexes

$$
0 \rightarrow \tilde{F}_{\bullet} \rightarrow \text { Cone }(\tilde{w}) \cdot(-1) \stackrel{\tilde{u}_{\bullet}(-1)}{\rightarrow} G_{\bullet}(-1) \rightarrow 0
$$

brings the exact sequence of homologies

$$
0 \rightarrow M \rightarrow \mathrm{H}_{-1}\left(\text { Cone }(\tilde{w})_{\bullet}\right) \rightarrow \Omega_{R}^{-1}\left(X^{\prime}\right) \rightarrow 0
$$

because $\mathrm{H}_{i}\left(\right.$ Cone $\left.(\tilde{w})_{\bullet}\right)=0(i \neq-1)$.

We claim that the above sequence (2.9) is the minimal finite projective hull of $M$. By definition, $\Omega_{R}^{-1}\left(X^{\prime}\right)$ is a stable Cohen-Macaulay module, so it suffices to show that $\mathrm{H}_{-1}\left(C_{\text {Cone }}(\tilde{w})_{\bullet_{0}}\right)$ is of finite projective dimension. Truncations $\sigma: \tilde{F}_{\bullet} \rightarrow \tau_{0} \tilde{F}_{\bullet}$ and $\tau: G_{\bullet} \rightarrow \tau_{0} G_{\bullet}$ induces a surjective chain map Cone $\left(\tilde{w}_{\bullet}\right)_{\bullet} \rightarrow$ Cone $\left(\tau_{0} \tilde{w}_{\bullet}\right)$. as in the diagram $(2.10)$

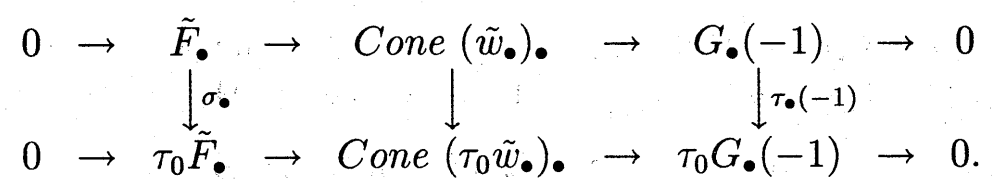

More precisely, we get the following commutative diagram whose rows and 
columns are exact.

$$
\begin{aligned}
& \downarrow \begin{array}{lll}
\vdots & \vdots \\
\downarrow & \vdots
\end{array} \\
& \text { Cone }(\tilde{w})_{0} \quad \text { Cone }\left(\tau_{0} \tilde{w}\right)_{0} \\
& 0+\| \quad=\cdots \\
& F_{M 1} \oplus G_{0} \quad F_{M 1} \oplus G_{0} \\
& \downarrow \quad\left(\begin{array}{cc}
d_{\tilde{F} 1} & \tilde{w}_{0} \\
0 & d_{G 0}
\end{array}\right) \downarrow \quad \downarrow\left(d_{\tilde{F} 1} \tilde{w}_{0}\right)
\end{aligned}
$$

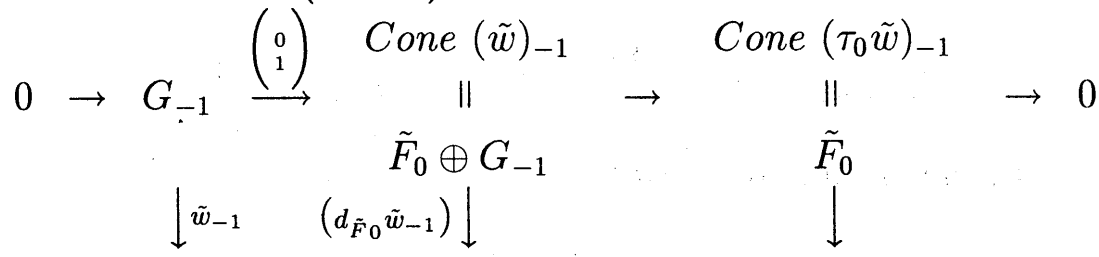

$$
\begin{aligned}
& P^{\prime}=\| \begin{array}{ll}
\| & 0
\end{array} \\
& \downarrow
\end{aligned}
$$

Giving the -1-th truncation and taking homology, we get the sequence

$$
\begin{gathered}
0 \rightarrow G_{-1} \rightarrow \quad \underset{2 \|}{\operatorname{Coker} d_{\text {Cone }(\tilde{w})_{0}}} \rightarrow \text { Coker } d_{\text {Cone }\left(\tau_{0} \tilde{w}\right)_{0}} \rightarrow 0 . \\
\mathrm{H}_{-1}\left(\operatorname{Cone}(\tilde{w})_{\bullet}\right) \oplus P^{\prime}
\end{gathered}
$$

Since the bottommost row of (2.10) induces the exact sequence of homologies of the complexes $(2.8), \mathrm{H}_{i}\left(\right.$ Cone $\left.\left(\tau_{0} \tilde{w}\right)_{\bullet}\right)=0$ for $i \neq-1$ and $\mathrm{H}_{-1}\left(\right.$ Cone $\left.\left(\tau_{0} \tilde{w}\right)_{\bullet}\right)=$ Coker $d_{\text {Cone }\left(\tau_{0} \tilde{w}\right)_{0}} \cong Y^{\prime}$. So the sequence $(2.11)$ tells us $\Omega_{R}^{1}\left(\mathrm{H}_{-1}\left(\right.\right.$ Cone $\left.\left.(\tilde{w})_{.}\right)\right) \stackrel{\text { st }}{\cong}$ $\Omega_{R}^{1}\left(Y^{\prime}\right)$, which implies that $\mathrm{H}_{-1}\left(\right.$ Cone $\left.(\tilde{w})_{\bullet}\right)$ is of finite projective dimension, hence is isomorphic to $Y^{M}$.

As

$$
\mathrm{H}_{i}\left(\text { Cone }(\tilde{w})_{\bullet}\right) \cong\left\{\begin{array}{ll}
Y^{M} & i=-1 \\
0 & i=\neq-1
\end{array},\right.
$$

and

$$
\mathrm{H}_{i}\left(\text { Cone }\left(\tau_{0} \tilde{w}\right)_{.}\right) \cong\left\{\begin{array}{ll}
Y^{\prime} & i=-1 \\
0 & i=\neq=-1
\end{array},\right.
$$

we have isomorphisms of complexes

$$
\begin{aligned}
\text { Cone }\left(\tilde{w}_{\bullet}\right) \bullet & \cong I_{M \bullet} \oplus T_{W \bullet}, \\
\text { Cone }\left(\tau_{0} \tilde{w}_{\bullet}\right) \bullet & \cong I^{\prime} \oplus T_{W^{\prime} \bullet}
\end{aligned}
$$


where $I_{M \bullet}(-1)$ is the minimal free resolution of $Y^{M}, I^{\prime} \bullet(-1)$ is that of $Y^{\prime}$, while $T_{W \bullet}$ and $T_{W^{\prime} \bullet}$ are the direct sums of trivial complexes.

Adding these split morphisms to the rightmost rectangular of (2.10), we have the following diagram.

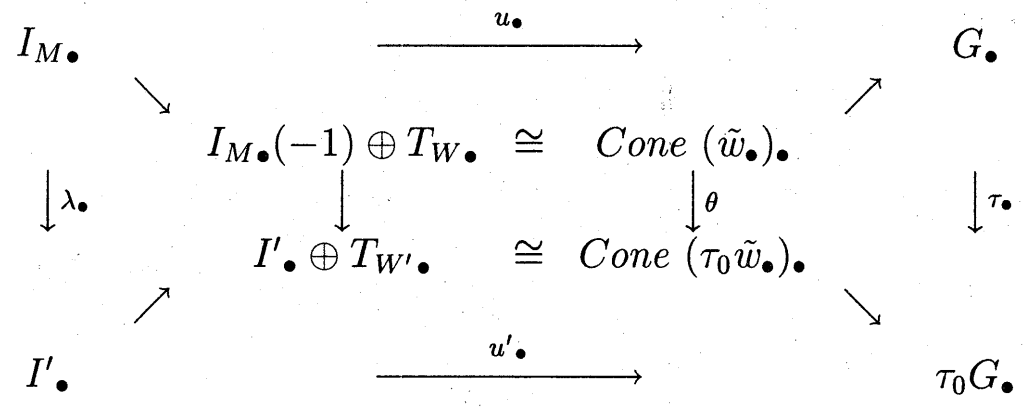

Notice that $u_{\bullet}$ here is nothing but $u_{M} \bullet$

We have the diagram

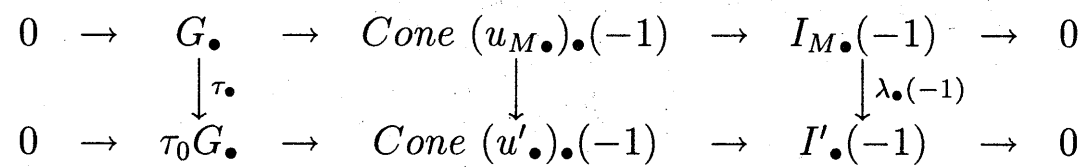

The topmost row of (2.13) induces the exact sequence

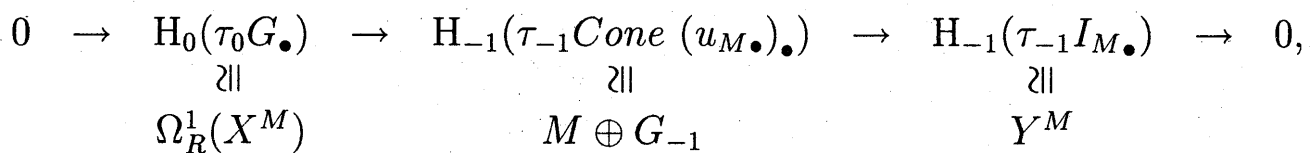

which is $(2.6)$ by definition.

The bottommost row of (2.13) induces the exact sequence

$$
\begin{aligned}
& 0 \rightarrow \mathrm{H}_{0}\left(\tau_{0} G_{\bullet}\right) \rightarrow \mathrm{H}_{-1}\left(\text { Cone }\left(u_{\bullet}^{\prime}\right)_{\bullet}\right) \rightarrow \mathrm{H}_{-1}\left(I_{\bullet}^{\prime}\right) \rightarrow 0, \\
& \begin{array}{lll}
\text { 2॥ } & \text { 2॥ } & \text { 2॥ } \\
X^{\prime} & M \oplus P^{\prime} & Y^{\prime}
\end{array}
\end{aligned}
$$

which is (2.8) from the basic property of the mapping cone.

It remains to explicitly describe the maps between each pair of modules in (2.6) and (2.8). We begin with $\lambda_{\bullet}$ and then the map Cone $\left(u_{M_{\bullet}}\right)_{\bullet}(-1) \rightarrow$ Cone $\left(u_{\bullet}^{\prime}\right)_{\bullet}(-1)$. Take a chain map $w_{\bullet}: G_{\bullet} \rightarrow F_{\bullet}$ as a composite of $\tilde{w}_{\bullet}$ and the natural map $\tilde{F}_{\bullet} \rightarrow F_{\bullet}$. We arrange bases of $G_{n}$ and $F_{n}$ so that $w_{\bullet}$ is described as

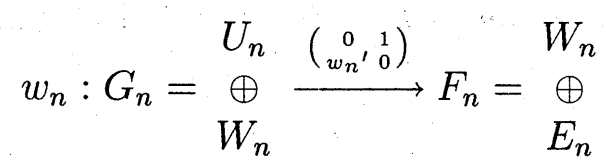

with $w_{n}^{\prime} \otimes k$ at each $n \geq 0$. According to these bases, put

$$
d_{G}=\underset{W}{U}\left(\begin{array}{cc}
U & W \\
g_{11} & g_{12} \\
g_{21} & g_{22}
\end{array}\right), \quad d_{F}=\begin{array}{cc}
W & E \\
E
\end{array}\left(\begin{array}{ll}
f_{11} & f_{12} \\
f_{21} & f_{22}
\end{array}\right),
$$


and $G_{-1}=U_{-1}$. Remember that $\tilde{w}_{0}=\left(\begin{array}{c}w_{0} \\ -\tilde{w}_{-1} d_{G 0}\end{array}\right)$, since $d_{\tilde{F} 0}=P^{\prime}\left(0^{F_{0} P^{\prime}}-1\right)$.

We look at how differentials are described with respect to the bases, first according to the central rectangular of the diagram (2.12). If $n \geq 0$, we have Cone $\left(\tilde{w}_{\bullet}\right)_{n}=$ Cone $\left(\tau_{0} \tilde{w}_{\bullet}\right)_{n}$ and $d_{\text {Cone }\left(\tilde{w}_{\bullet}\right)_{n+1}}=d_{\text {Cone }\left(\tau_{0} \tilde{w}_{\bullet}\right)_{n+1}}$, in which cases we may change the basis as follows:

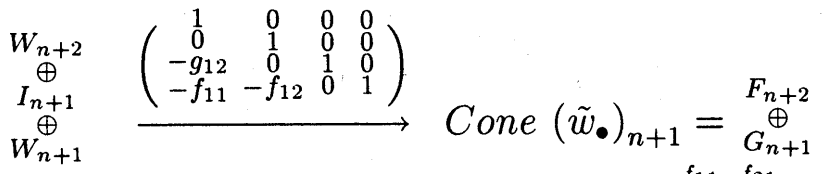

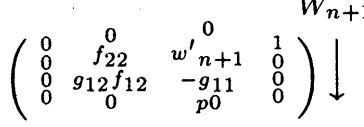

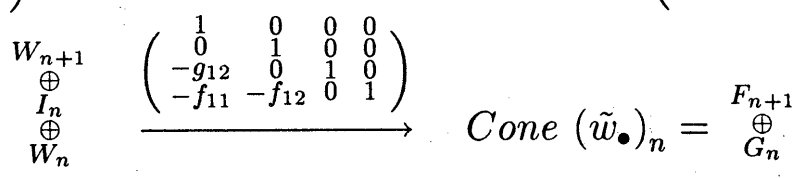

$$
\begin{aligned}
& \downarrow\left(\begin{array}{cccc}
f_{11} & f_{21} & 0^{0} & \\
f_{21} & f_{22} & w_{n+1}^{\prime} & 1 \\
0 & 0 & -g_{11} & 0 \\
0 & 0 & -g_{21} & -g_{22}
\end{array}\right)
\end{aligned}
$$

And this diagram goes to the next one as $n=-1$ :

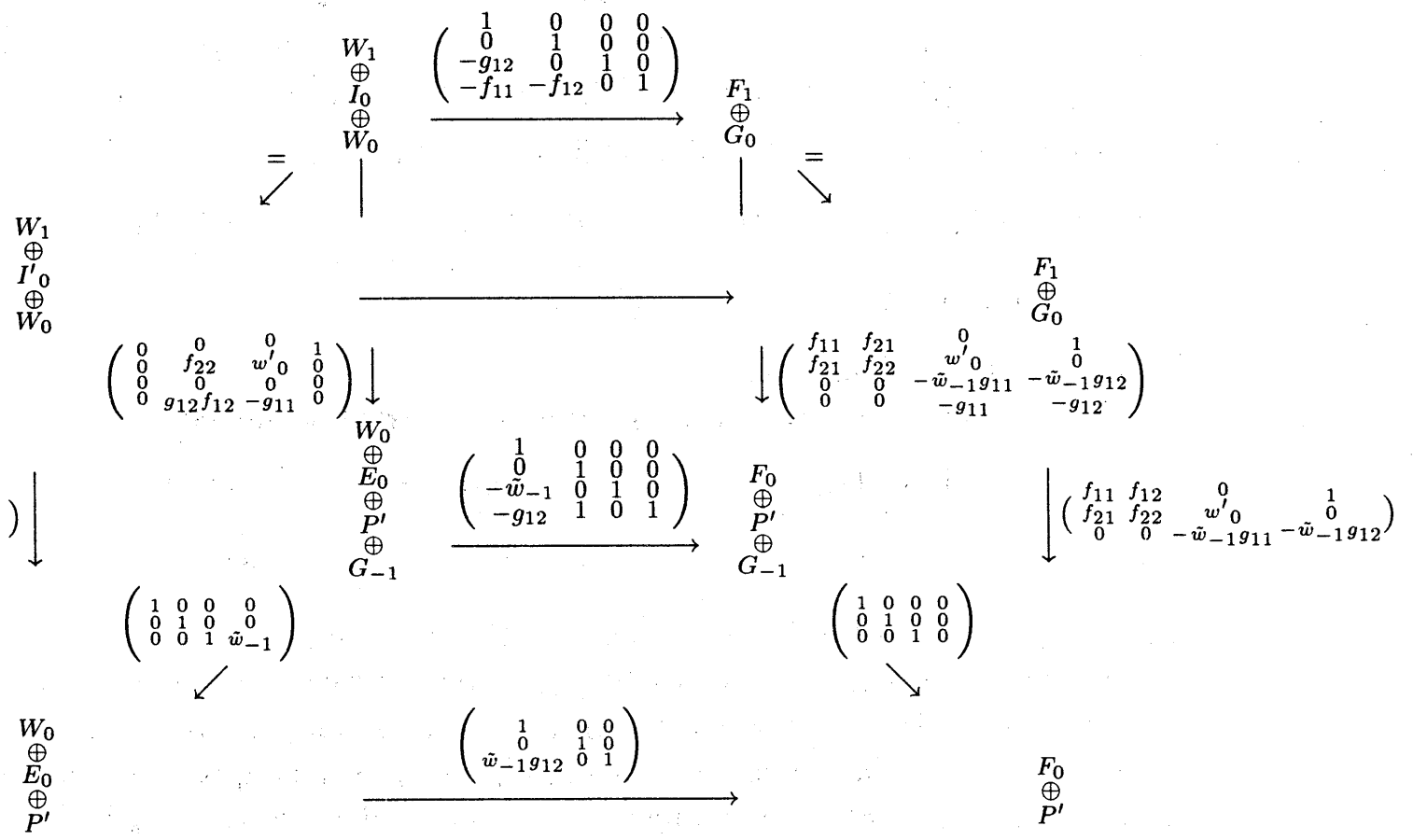

Thus we obtain the complex $\left(I_{M_{\bullet}}, d_{I_{M}}\right)$ and the chain map $u_{M \bullet}: I_{M \bullet} \rightarrow$ $G_{\bullet}$;

$$
\begin{aligned}
& u_{M n}=(-1)^{n}\left(\begin{array}{cc}
0 & 1 \\
-f_{12} & 0
\end{array}\right) \\
& \begin{array}{l}
I_{M n}=E_{n+1} \oplus U_{n} \quad G_{M n}=U_{n} \oplus W_{n} \\
\left(\begin{array}{cc}
f_{22} & w_{n}^{\prime} \\
g_{12} f_{12} & g_{11}
\end{array}\right) \downarrow \\
I\left(\begin{array}{ll}
g_{11} & g_{21} \\
g_{12} & g_{22}
\end{array}\right)
\end{array} \\
& I_{M n-1}=E_{n} \oplus U_{n-1} \quad \longrightarrow \quad G_{M n-1}=U_{n-1} \oplus W_{n-1}
\end{aligned}
$$


for $n \geq-1$ where $w_{-1}=0$. While

$$
I_{n}^{\prime}=I_{M n}, \quad d_{I^{\prime} n}=d_{I_{M n}}, \lambda_{n}=\mathrm{id}_{I_{n}^{\prime}}, \quad u_{n}^{\prime}=u_{M n}
$$

for $n \geq 0$, and

$$
\begin{aligned}
& I_{-1}^{\prime}=E_{0} \oplus P^{\prime}, \quad d_{I^{\prime} 0}=\stackrel{E_{0}}{P^{\prime}}\left(\begin{array}{cc}
E_{1} & U_{0} \\
f_{22} & w_{0}^{\prime} \\
\tilde{w}_{-1} g_{12} f_{12} & \tilde{w}_{-1} g_{11}
\end{array}\right) \\
& \lambda_{-1}=\stackrel{E_{0}}{P}\left(\begin{array}{cc}
E_{0} & U_{-1} \\
1 & 0 \\
0 & \tilde{w}_{-1}
\end{array}\right), \quad u_{-1}^{\prime}=0
\end{aligned}
$$

We are now on the next stage to look at the mapping cones of $u_{M}$ and $u_{\bullet}^{\prime}$. Similarly as above, we have a diagram

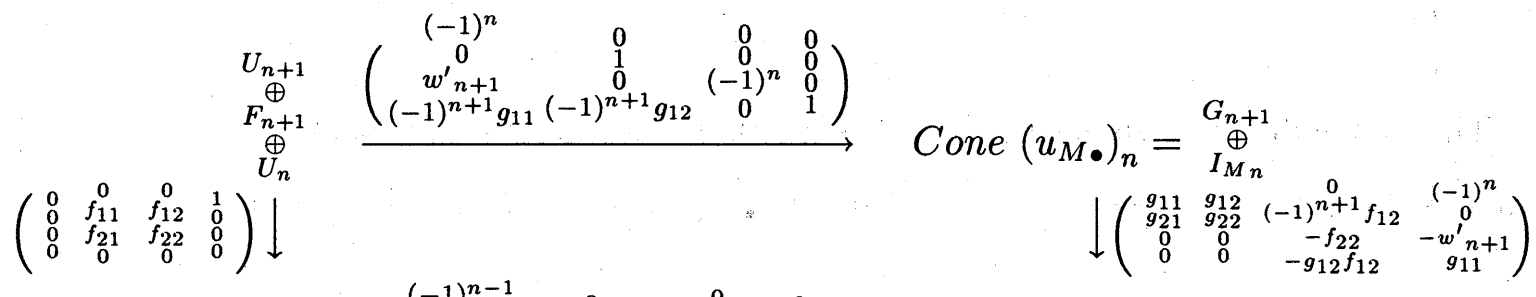

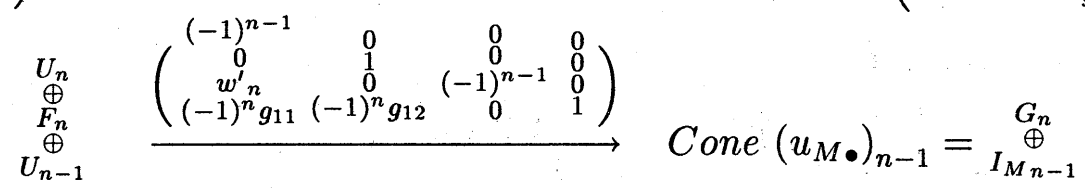

for $n \geq 0$ where Cone $\left(u_{M_{\bullet}}\right)_{n}=$ Cone $\left(u_{\bullet}^{\prime}\right)_{n}$ and $d_{\text {Cone }\left(u_{M} \bullet\right)_{n}}=d_{\text {Cone }\left(u^{\prime} \bullet\right)_{n}}$, 
whose lower part for $n=0,-1$ is

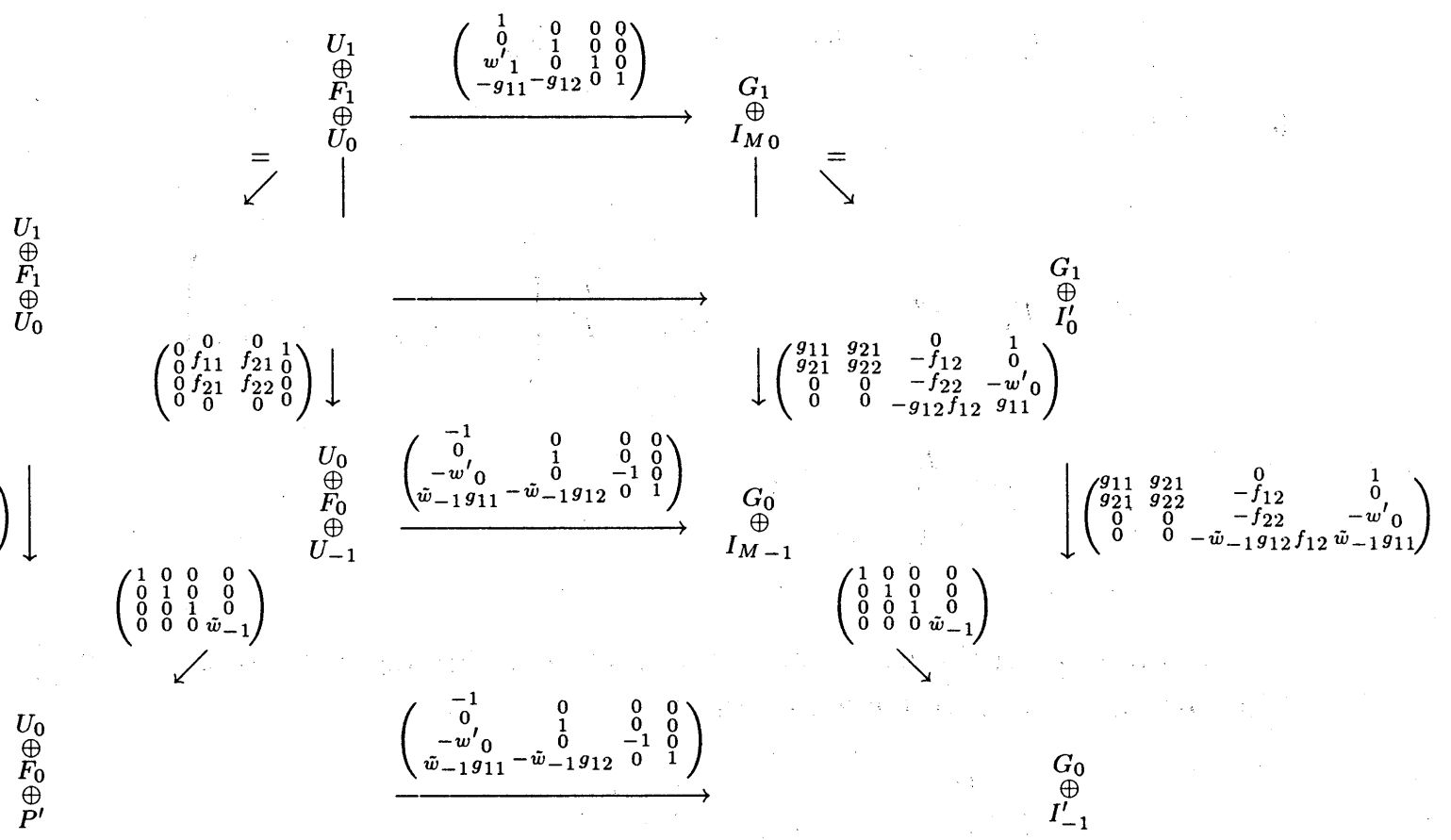

The above diagram says that (2.13) is modified through isomorphisms as :

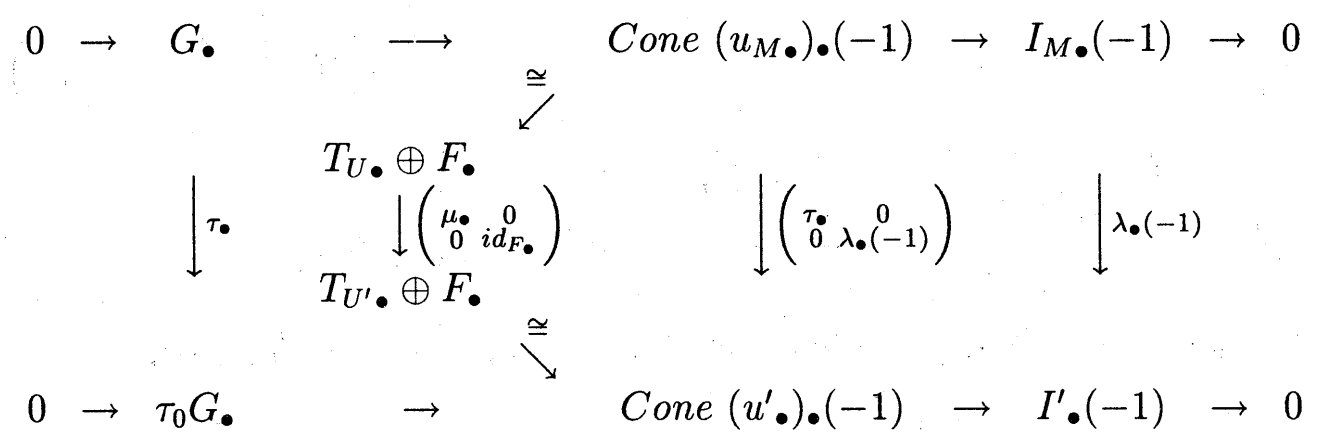

where $\mu_{\bullet}: T_{U_{\bullet}} \rightarrow T_{U^{\prime}}$ is the chain map between trivial complexes;

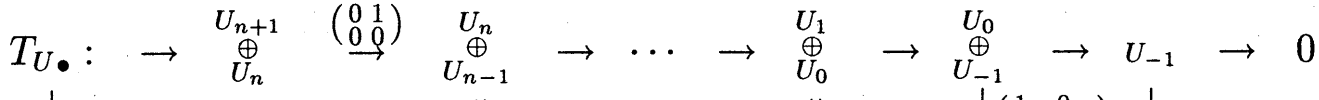

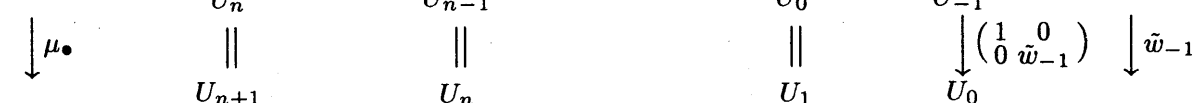

$$
\begin{aligned}
& T_{U^{\prime} \bullet}: \rightarrow \begin{array}{c}
U_{n+1} \\
\underset{U_{n}}{\oplus}
\end{array} \rightarrow \underset{U_{n-1}}{U_{n}} \rightarrow \cdots \rightarrow \underset{U_{0}}{\stackrel{U_{1}}{\oplus}} \rightarrow \underset{P^{\prime}}{\stackrel{\oplus}{U_{0}}} \rightarrow P^{\prime} \rightarrow 0 .
\end{aligned}
$$

Consequently, we have a commutative diagram

$$
\begin{aligned}
& 0 \rightarrow \Omega_{1}^{R}\left(X^{M}\right) \rightarrow M \oplus G_{-1} \rightarrow Y^{M} \rightarrow 0
\end{aligned}
$$

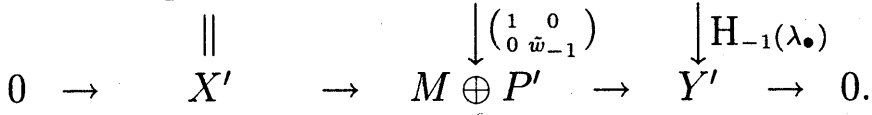

(q.e.d for Theorem 2.2.) 
Theorem 2.3 The minimal original extension of an $R$-module $M$ is unique up to isomorphism. In other word, if two original extensions of $M ; 0 \rightarrow X \rightarrow$ $M \oplus P \rightarrow Y \rightarrow 0$ and $0 \rightarrow X^{\prime} \rightarrow M \oplus P^{\prime} \rightarrow Y^{\prime} \rightarrow 0$ are both minimal, linear maps $a, b$ and $c$ in the diagram (2.4) are isomorphisms. The minimal original extension of $M$ is, after adding some free summand, of the form

$$
0 \rightarrow \Omega_{R}^{1}\left(X^{M}\right) \rightarrow M \oplus G_{-1} \rightarrow Y^{M} \rightarrow 0
$$

where $G_{-1} \rightarrow X^{M}$ is the minimal projective cover.

proof) From the condition 3) of the minimal original extension, there exist homomorphisms $a, b, c, a^{\prime}, b^{\prime}$, and $c$ that makes the next commutative diagram:

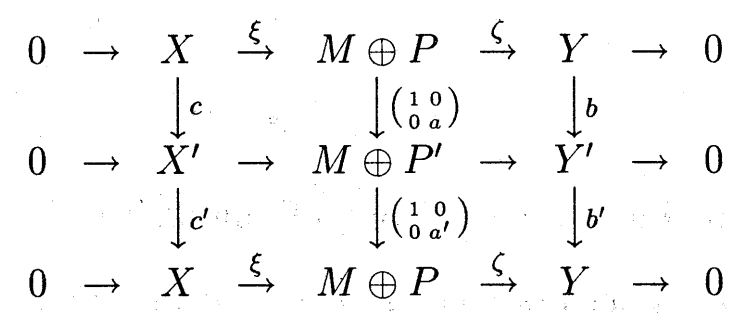

We shall show that $a^{\prime} a: P \rightarrow P$ is an isomorphism. Reviewing the proof of Theorem 2.2, we may take $c$ and $c^{\prime}$ as an identity map of $X$. We have $\xi^{P}=$ $a^{\prime} a \xi^{P}$ from the diagram above where $\xi^{P}$ is a composite $\xi^{P}: X \stackrel{\xi}{\rightarrow} M \oplus P \rightarrow P$. The minimal cover $G_{0} \stackrel{d_{G 0}}{\rightarrow} X$ induces a homomorphism $x^{P}$ as

$$
\begin{aligned}
& G_{\downarrow_{G 0}} \stackrel{x^{P}}{\rightarrow} \quad P \\
& X \stackrel{\xi^{P}}{\rightarrow} P .
\end{aligned}
$$

This $x^{P}$ has the same property $x^{P}=a^{\prime} a x^{P}$, which is observed as follows. With respect to matrix representation $a^{\prime} a=\left(a_{i j}\right)_{1 \leq i, j \leq \mathrm{rk}(P)}$, and $x^{P}=\left(x_{k l}\right)_{1 \leq k \leq \mathrm{rk}(P), 1 \leq l \leq \operatorname{rk}\left(G_{0}\right)}$, the above equation means $\left(a^{\prime} a x^{P}\right)_{i j}=x^{P}{ }_{i j}$, that is,

$$
\sum_{k=1}^{\mathrm{rk}(P)} a_{i k} x_{k j}=x_{i j}
$$

for $1 \leq i \leq \operatorname{rk}(P), 1 \leq j \leq \operatorname{rk}\left(G_{0}\right)$. Now suppose that $a^{\prime} a$ is not an isomorphism. Then it has at least one row, say, the first row, whose all entries belong to the maximal ideal $\mathfrak{m}$. We have

$$
\left(1-a_{11}\right) x_{1 j}=\sum_{k=1}^{\operatorname{rk}(P)} a_{1 k} x_{k j}
$$

for $1 \leq j \leq \operatorname{rk}(P)$ with $\left(1-a_{11}\right)$ a unit, which implies that $x^{P}$ has a zero row after some row-transformations.

On the other hand, it is easy to see the equivalence of these conditions: 
1) A common summand split off through $\zeta$ from $X$ and $Y$.

2) There exists a split epimorphism $s: P \rightarrow R$ such that $s \xi^{P}=0$.

3) There exists a split epimorphism $s: P \rightarrow R$ such that $s x^{P}=0$.

4) After some row-transformations, $x^{P}$ contains a zero-row.

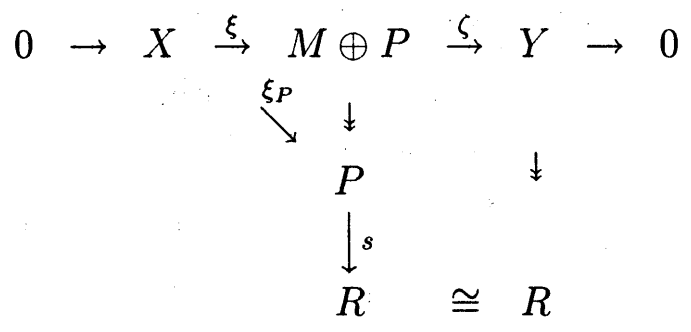

So we get a contradiction to the condition of minimality. (q.e.d)

Remark 2.4 The minimal original extension of the direct sum $M \oplus N$ of modules is the direct sum of the minimal original extension of $M$ and that of $N$.

We refresh our memory on our attitude to regard an element of the module $\operatorname{Ext}_{R}^{1}(M, N)$ as a chain map. More precisely, an element $\theta \in \operatorname{Ext}_{R}^{1}(M, N)$ as an exact sequence started from $N$ and ended with $M$ corresponds to a chain map $\theta_{\bullet} \in \operatorname{Hom}_{R}\left(F_{\bullet}, G_{\bullet}\right)$ of degree zero where $F_{\bullet}(-1) \rightarrow M$ and $G_{\bullet} \rightarrow N$ are the minimal free resolutions.

First take a chain map $\theta_{\bullet} \in \operatorname{Hom}_{R}\left(F_{\bullet}, G_{\bullet}\right)$, then the exact sequence of the complexes

$$
0 \rightarrow G_{\bullet} \rightarrow \text { Cone }\left(\theta_{\bullet}\right) \bullet(-1) \rightarrow F_{\bullet}(-1) \rightarrow 0
$$

induces the exact sequence of homologies

$$
0 \rightarrow N \rightarrow \text { Coker } d_{\text {Cone }\left(\theta_{\bullet}\right)_{0}} \rightarrow M \rightarrow 0
$$

which is the corresponding exact sequence $\theta \in \operatorname{Ext}_{R}^{1}(M, N)$.

Conversely, for an exact sequence

$$
\theta: 0 \rightarrow N \stackrel{\bar{\xi}}{\rightarrow} X \rightarrow M \rightarrow 0
$$

take a chain map $\xi_{\bullet}: G_{\bullet} \rightarrow I_{\bullet}$ as $\mathrm{H}_{0}\left(\xi_{\bullet}\right)=\bar{\xi}$ with the minimal free resolutions $G_{\bullet} \rightarrow N$ and $I_{\bullet} \rightarrow X$. Let $F_{\bullet}(-1) \rightarrow M$ be the minimal free resolution. As for the exact sequence of the complexes

$$
0 \rightarrow I_{\bullet} \rightarrow \text { Cone }\left(\xi_{\bullet}\right) \bullet(-1) \rightarrow G_{\bullet}(-1) \rightarrow 0
$$


take the chain map $\theta_{\bullet}(-1): F_{\bullet}(-1) \rightarrow G_{\bullet}(-1)$ so that the composite of the quasi-isomorphism Cone $\left(\xi_{\bullet}\right)_{\bullet}(-1) \rightarrow F_{\bullet}(-1)$ and $\theta_{\bullet}(-1)$ is the natural epimorphism Cone $\left(\xi_{\bullet}\right)_{\bullet}(-1) \rightarrow G_{\bullet}(-1)$. Then this $\theta_{\bullet}$ is the chain map that corresponds to the given exact sequence $\theta$. And from the fundamental property. of mapping cone, we easily see that $\theta_{\bullet}$ goes back to $\theta$ via the procedure above.

From now on, we use the notation $\theta_{\bullet}$ to represents an element of $\operatorname{Ext}_{R}^{1}(M, N)$ and do not distinguish a chain map from the corresponding exact sequence. And if $N=\Omega_{R}^{1}\left(N^{\prime}\right)$, for $f_{\bullet} \in \operatorname{Hom}_{R}\left(F_{\bullet}, G_{\bullet}^{\prime}\right)$ with the minimal free resolution $G^{\prime}{ }_{\bullet}(-1) \rightarrow N^{\prime}$, we define an element $r \operatorname{tr}\left(f_{\bullet}\right)_{\bullet} \in \operatorname{Hom}_{R}\left(F_{\bullet}, \tau_{0} G^{\prime} \bullet=\right.$ $\left.G_{\bullet}\right)$ with $r \operatorname{tr}(f)_{i}:=f_{i} \quad(i \geq 0)$ and $\operatorname{rtr}(f)_{-1}=0$.

The minimal finite projective hull of $M:=\Omega_{R}^{1}(N)$ is of the form

$$
0 \rightarrow M \rightarrow \Omega_{R}^{1}\left(Y^{N}\right) \oplus W_{N 0} \rightarrow \Omega_{R}^{1}\left(X^{N}\right) \rightarrow 0
$$

which gives an original extension of $M$

$$
0 \rightarrow \Omega_{R}^{2}\left(X^{N}\right) \rightarrow M \oplus U_{N_{0}} \oplus W_{N_{0}} \rightarrow Y^{N} \oplus W_{N_{0}} \rightarrow 0
$$

where $W_{N_{0}}$ and $U_{N_{0}}$ are free modules and $\operatorname{rk}\left(W_{N_{0}} \oplus U_{N_{0}}\right)$ equals to a minimal number of generators of $\Omega_{R}^{1}\left(X^{N}\right)$. The minimal original extension of $M$ is thus

$$
0 \rightarrow \Omega_{R}^{2}\left(X^{N}\right) \rightarrow M \oplus U_{N 0} \rightarrow Y^{N} \rightarrow 0 .
$$

As the most extreme case, the minimal original extension of a stable CohenMacaulay module $C$ is

$$
0 \rightarrow C=C \rightarrow 0 \rightarrow 0 .
$$

However, as elements of $\operatorname{Ext}_{R}^{1}(-,-)$, we can ignore those differences by split exact sequences. In other word, we are not interested in original extensions that are non-minimal for lack of the property 1) and 2) of the definition. Alternatively, our next concern is about the non-trivial non-minimal original extension which differs from the minimal one by the property 3 ).

Any non-minimal Cohen-Macaulay approximation or finite projective hull is the direct sum of the minimal one and some trivial complex. Although it is not the case for non-minimal original extension as seen in Example 2.8. Let $0 \rightarrow X \rightarrow M \oplus P \rightarrow Y \rightarrow 0$ be an original extension of a stable $R$-module $M$ that is not necessarily minimal. We observe that

$$
X \cong \Omega_{R}^{1}\left(X^{M}\right) \text { up to free summands, }
$$

and

$$
0 \rightarrow G_{M-1} \rightarrow Y^{M} \oplus P \rightarrow Y \rightarrow 0
$$

where $G_{M-1} \rightarrow X^{M}$ is the minimal projective cover. from the argument in the proof of Theorem 2.2. 
Lemma 2.5 For a module $Y$ with finite projective dimension, the following are equivalent.

1)

$$
\operatorname{Ext}_{R}^{1}(Y, R)=0
$$

2) For any stable Cohen-Macaulay module $X$, each non-zero element of $\operatorname{Ext}_{R}^{1}(Y, X)$ is the minimal original extension of a stable module.

proof) It suffices to prove for a stable $Y$.

To see the implication from 1) to 2), suppose the contrary; let $0 \rightarrow X \rightarrow$ $M \oplus P \rightarrow Y \rightarrow 0$ be a non-minimal original extension of a stable module $M$. Then we have a non-split exact sequence (2.15) $0 \rightarrow G_{-1} \rightarrow Y^{M} \oplus P \rightarrow Y \rightarrow 0$, which contradicts to the condition 1).

Stronger than the other implication, we show the next statement: If $\operatorname{Ext}_{R}^{1}(Y, R) \neq 0$, for any stable Cohen-Macaulay module $X$ with the property $\operatorname{Hom}_{R}(Y, X) \neq 0, \operatorname{Ext}_{R}^{1}(Y, X)$ contains a non-trivial non-minimal original extension. Notice that if $\operatorname{Hom}_{R}\left(Y, X^{\prime}\right)=0$ for any stable Cohen-Macaulay module $X^{\prime}$, then $\operatorname{Ext}_{R}^{1}(Y, X)=0$ for any stable Cohen-Macaulay module $X$ and there is nothing to prove. To show this, we have only to see the epimorphism $\operatorname{Hom}_{R}\left(\Omega_{R}^{-1}(X), P\right) \rightarrow \operatorname{Ext}_{R}^{1}(Y, X)$ applying $\operatorname{Hom}_{R}(Y$,$) to$ $0 \rightarrow X \rightarrow P \rightarrow \Omega_{R}^{-1}(X) \rightarrow 0$ with a free module $P$.

So we assume that $\operatorname{Hom}_{R}(Y, X) \neq 0$ for a stable Cohen-Macaulay module $X$. Take a non-zero element $f \in \operatorname{Hom}_{R}(Y, X)$, then together with the minimal projective cover $P \rightarrow$ Coker $f$ we have an epimorphism $Y \oplus P \rightarrow X$ whose kernel we call $M$;

$$
0 \rightarrow M \rightarrow Y \oplus P \rightarrow X \rightarrow 0
$$

While the hypothesis $\operatorname{Ext}_{R}^{1}(Y, R) \neq 0$ gives us the non-split exact sequence

$$
0 \rightarrow Q \rightarrow Y^{\prime} \rightarrow Y \rightarrow 0
$$

with a free module $Q$. Obviously $Y^{\prime}$ has a finite projective dimension. 
We get the pull-back diagram from the sequences (2.16) and (2.17)

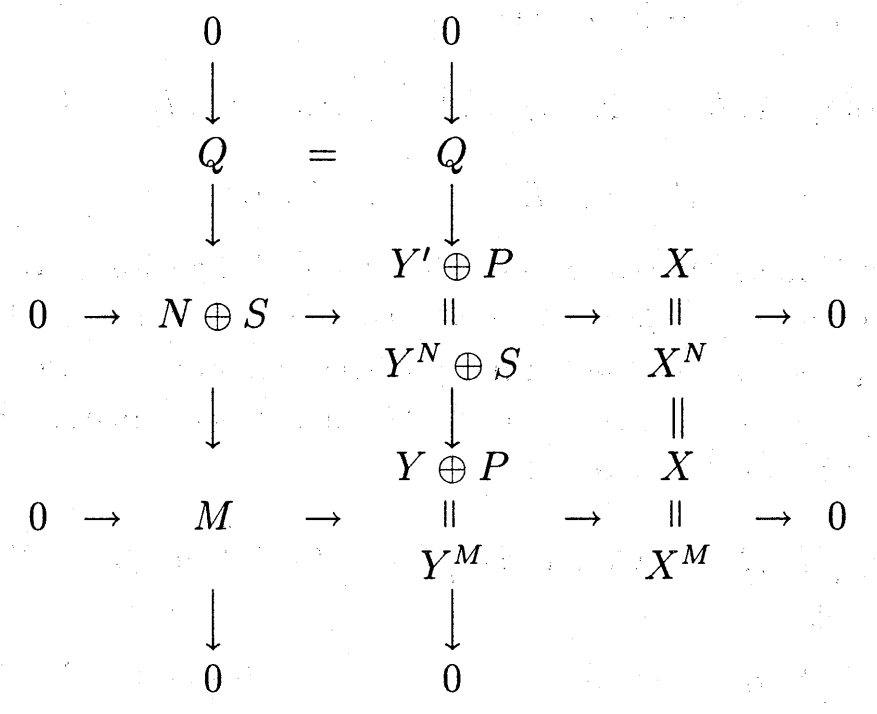

where $N$ is a stable module and $S$ is a free module. The minimal original extension (added some free modules) of $N \oplus S$ and the sequence (2.17) make another pull-back diagram:

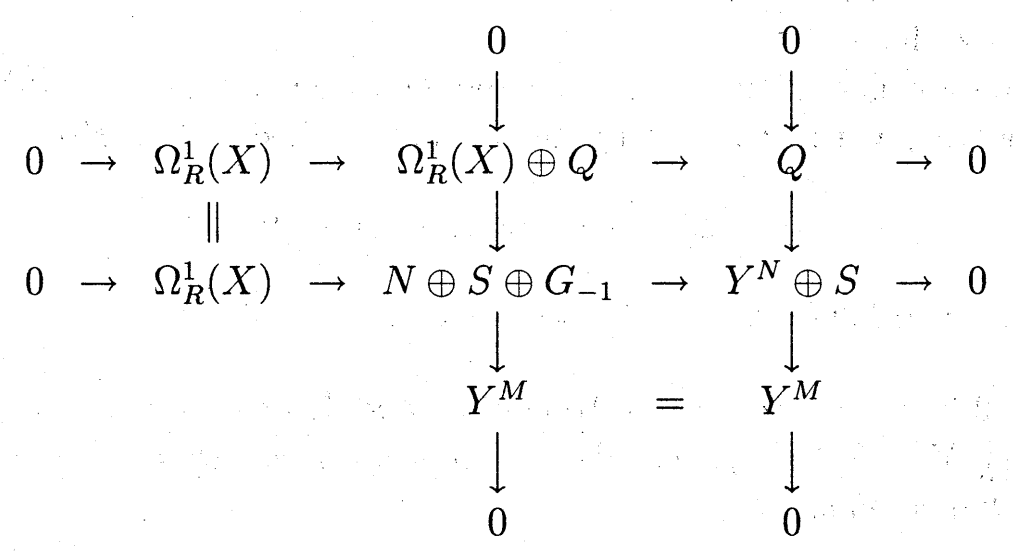

Here the middle column is an original extension of $N \oplus S$ that is non-trivially non-minimal because the rightmost column (2.17) does not split. (q.e.d.)

Lemma 2.6 Let $M$ be an indecomposable Cohen-Macaulay module with codimension $r>1$. Let $\theta: 0 \rightarrow \Omega_{R}^{1}\left(X^{M}\right) \rightarrow L \oplus P \rightarrow Y^{M} \rightarrow 0$ be a non-trivial element of $\operatorname{Ext}_{R}^{1}\left(Y^{M}, \Omega_{R}^{1}\left(X_{M}\right)\right)$ where $L$ is a stable module. Then, $L \cong M$.

proof) Lemma 2.5 tells us $\theta$ is the minimal original extension of $L$, which implies $Y^{L} \oplus P \cong Y^{M} \oplus G_{-1}$ hence $Y^{L} \cong Y^{M}=: Y$ since $P \cong G_{-1}$ from $X^{L} \cong X^{M}=: X$. The sequence $0 \rightarrow M \rightarrow Y \rightarrow X \rightarrow 0$ induces

$$
\operatorname{Hom}_{R}(Y, R) \cong \operatorname{Hom}_{R}(X, R) \text {, }
$$




$$
\operatorname{Ext}_{R}^{i}(Y, R) \cong \operatorname{Ext}_{R}^{i}(M, R) . \quad(i \neq r, 0)
$$

While $0 \rightarrow L \rightarrow Y \rightarrow X \rightarrow 0$ induces

$$
\begin{gathered}
0 \rightarrow \operatorname{Hom}_{R}(X, R) \rightarrow \operatorname{Hom}_{R}(Y, R) \rightarrow \operatorname{Hom}_{R}(L, R) \rightarrow 0, \\
\operatorname{Ext}_{R}^{i}(L, R)=0 . \quad(i \neq r, 0)
\end{gathered}
$$

If $L$ is also a Cohen-Macaulay module with codimension $r$, or equivalently $\operatorname{Hom}_{R}(L, R)=0, L^{\vee} \cong \operatorname{Ext}_{R}^{r}(Y, R) \cong M^{\vee}$ therefore $L \cong L^{\vee \vee} \cong M^{\vee \vee} \cong M$.

Putting $F_{L \bullet} \rightarrow L$ as the minimal free resolution, we have $L^{*}=$ $\Omega_{R}^{r+1}\left(\operatorname{Coker}\left(d_{F_{L} r}\right)^{*}\right)$ from (2.20). While the exact sequence with a maximal Cohen-Macaulay module at the tail

$$
\begin{aligned}
& 0 \rightarrow \operatorname{Ext}_{R}^{r}(L, R) \rightarrow \operatorname{Coker}\left(d_{F_{L r}}\right)^{*} \rightarrow F_{L r}{ }^{*} / \operatorname{Ker}\left(d_{F_{L r+1}}\right)^{*} \rightarrow 0 \\
& \text { 2II 2II } \\
& \operatorname{Ext}_{R}^{r}(Y, R) \quad \operatorname{Im}\left(d_{F_{L r+1}}\right)^{*} \\
& \text { 211 2॥ } \\
& M^{\vee} \quad \operatorname{Ker}\left(d_{F_{L r+2}}\right)^{*}
\end{aligned}
$$

implies depth Coker $\left(d_{F_{L r}}\right)^{*} \geq \operatorname{dim} R-r$, hence $L^{*}$ is a maximal CohenMacaulay module.

Now the sequence (2.19) is an exact sequence of maximal Cohen-Macaulay modules together with (2.18), it remains exact applied ( $)^{*}:=\operatorname{Hom}_{R}(, R)$;

$$
0 \rightarrow L^{* *} \rightarrow Y^{* *} \cong X \rightarrow X \rightarrow 0 .
$$

It follows $L^{* *}=0$ hence $L^{*} \cong L^{* * *}=0$. (q.e.d.)

Corollary 2.7 If $M$ is a Cohen-Macaulay module of codimension $r>1, R$ module $\operatorname{Ext}_{R}^{1}\left(Y^{M}, \Omega_{1}^{R}\left(X^{M}\right)\right)$ has the minimal original extension $\operatorname{rtr}\left(u_{M}\right.$. as a unique nontrivial element.

proof) It follows from Lemma 2.5 and Lemma 2.6 altogether.

Example 2.8 Let $R:=k[[x, y]] /(x y)$, and $M:=k$. We have

$$
\begin{gathered}
\cdots \rightarrow R^{2} \stackrel{\left(\begin{array}{ll}
x & 0 \\
0 & y
\end{array}\right)}{\longrightarrow} R^{2} \stackrel{\left(\begin{array}{ll}
y & 0 \\
0 & x
\end{array}\right)}{\longrightarrow} R^{2} \stackrel{(x y)}{\longrightarrow} R \rightarrow k \rightarrow 0, \\
\cdots \rightarrow R^{2} \stackrel{\left(\begin{array}{ll}
x & 0 \\
0 & y
\end{array}\right)}{\longrightarrow} R^{2} \stackrel{\left(\begin{array}{ll}
y & 0 \\
0 & x
\end{array}\right)}{\longrightarrow} R^{2} \rightarrow X^{M} \rightarrow 0, \\
0 \rightarrow R \stackrel{\left(\begin{array}{l}
y \\
x
\end{array}\right)}{\longrightarrow} Y^{M} \rightarrow 0 .
\end{gathered}
$$


Taking a finite projective dimensional module $Y^{\prime}$ as

$$
\begin{aligned}
0 \rightarrow & R \stackrel{\left(\begin{array}{l}
y \\
x
\end{array}\right)}{\rightarrow} R^{2} \rightarrow Y^{M} \rightarrow 0 \\
& R_{\left(\begin{array}{ll}
y & 0 \\
0 & x
\end{array}\right)} \downarrow_{\lambda}
\end{aligned}
$$

we get

$$
\begin{aligned}
& 0 \rightarrow \Omega_{R}^{1}\left(X^{M}\right) \rightarrow M \oplus R^{2} \rightarrow Y^{M} \rightarrow 0
\end{aligned}
$$

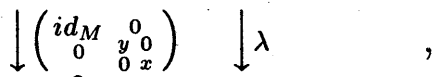

$$
\begin{aligned}
& 0 \rightarrow \Omega_{R}^{1}\left(X^{M}\right) \rightarrow M \oplus R^{2} \stackrel{0 x}{\rightarrow} Y^{\prime} \rightarrow 0
\end{aligned}
$$

where the second row is a non-minimal original extension of $M$ that is not a direct summand of the first row.

Lemma 2.9 For an $R$-module $Y$ with a finite projective dimension, assume that $Y^{*}=\operatorname{Hom}_{R}(Y, R)$ is Cohen-Macaulay. Then for any Cohen-Macaulay module $X$ and each element $\theta: 0 \rightarrow X \rightarrow L \oplus P \rightarrow Y$ of $\operatorname{Ext}_{R}^{1}(Y, X)$, we have a homomorphism $u_{L}: Y \rightarrow X$ such that

$$
\begin{aligned}
& \operatorname{Ext}_{R}^{1}\left(Y, u_{L}^{* *}\right): \operatorname{Ext}_{R}^{1}\left(Y, Y^{* *}\right) \rightarrow \operatorname{Ext}_{R}^{1}(Y, X) \\
& r t r\left(u_{M}\right) \\
& \theta \text {. }
\end{aligned}
$$

where $M$ is the module $0 \rightarrow M \rightarrow Y \rightarrow Y^{* *} \rightarrow 0$.

Lemma 2.10 Let $M$ be an indecomposable module with $\operatorname{Hom}_{R}(M, R)=0$, $\operatorname{Ext}_{R}^{1}(M, R)=0$. Then for any stable Cohen-Macaulay module $X$ and $\theta_{\bullet} \in$ $\operatorname{Ext}_{R}^{1}\left(Y^{M}, X\right)$, there exists a linear map $\phi_{X}: \Omega_{R}^{1}\left(X^{M}\right) \rightarrow X$ such that the induced homomorphism $\operatorname{Ext}_{R}^{1}\left(Y^{M}, \phi_{X}\right): \operatorname{Ext}_{R}^{1}\left(Y^{M}, \Omega_{R}^{1}\left(X^{M}\right)\right) \rightarrow \operatorname{Ext}_{R}^{1}\left(Y^{M}, X\right)$ sends $\operatorname{rtr}\left(u_{M_{\bullet}}\right)$ to $\theta_{\bullet}$.

proof) Let $\theta$. be

$$
\theta_{.}: 0 \rightarrow X \rightarrow N \oplus P \rightarrow Y^{M} \rightarrow 0
$$

with a stable module $N$ and a free module $P$.

The hypothesis gives us

$$
\begin{gathered}
\operatorname{Hom}_{R}\left(Y^{M}, R\right) \cong \operatorname{Hom}_{R}\left(X^{M}, R\right) \\
\operatorname{Ext}_{R}^{1}\left(Y^{M}, R\right)=0
\end{gathered}
$$

By Lemma 2.5, the equation (2.22) tells us $\theta_{\bullet}$ is the minimal original extension of $N$, in other word, $\theta_{\bullet} \cong \operatorname{rtr}\left(u_{N_{\bullet}}\right)$. We have $X \cong \Omega_{R}^{1}\left(X^{N}\right)$ and $Y^{M} \cong Y^{N}=$ : $Y$. On the $R$-dual ()$^{*}:=\operatorname{Hom}_{R}(, R)$ of the minimal finite projective hull of $N$

$$
0 \rightarrow\left(X^{N}\right)^{*} \rightarrow Y^{*} \rightarrow N^{*} \rightarrow 0
$$


taking an $R$-dual again, we have

$$
0 \rightarrow N^{* *} \rightarrow X^{M} \stackrel{\phi^{\prime}}{\rightarrow} X^{N} \rightarrow \operatorname{Ext}_{R}^{1}\left(N^{*}, R\right) \rightarrow 0
$$

from (2.22). To describe the chain map $\phi_{X_{\bullet}}: G_{M} \rightarrow G_{N_{\bullet}}$ induced by $\phi^{\prime}$, let $I_{.}(-1)$ be the minimal free resolution of $Y$, and consider the diagram:

$$
\begin{aligned}
& I_{0}{ }^{*} \leftarrow I_{-1}{ }^{*} \longleftarrow{ }_{\nwarrow} \longleftarrow G_{M-2}{ }^{*}
\end{aligned}
$$

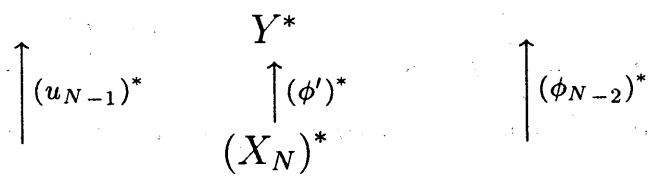

$$
\begin{aligned}
& \left(G_{N-1}\right)^{*} \stackrel{\left(d_{G_{N-1}}\right)^{*}}{\longleftarrow}\left(G_{N-2}\right)^{*}
\end{aligned}
$$

We have the commutativity

$$
\left(u_{N-1}\right)^{*}\left(d_{G_{N-1}}\right)^{*}=\Delta\left(\phi_{X-2}\right)^{*} .
$$

From another commutative diagram

$$
\begin{aligned}
& \left(G_{M-1}\right)^{*} \stackrel{\left(d_{G_{M-1}}\right)^{*}}{\nwarrow}\left(G_{M-2}\right)^{*}
\end{aligned}
$$

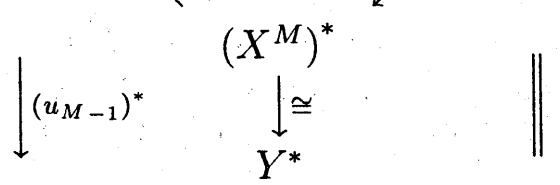

$$
\begin{aligned}
& I_{-1}{ }^{*} \longleftarrow \Delta G_{M-2}{ }^{*},
\end{aligned}
$$

we have $\Delta=\left(d_{G_{M-1}} u_{M-1}\right)^{*}$. By the substitution of this, (2.23) is modified into $d_{G_{N-1}}\left(\phi_{N-1} u_{M-1}-u_{N-1}\right)=0$, which means $\phi_{N_{\bullet}} u_{M \bullet}=u_{N}$ up to homotopy. (q.e.d.)

\section{Cohen-Macaulay approximations}

In this section, we discuss Cohen-Macaulay approximation within the framework of the theory of triangulated categories. Let us begin with the epimorphism $u_{M}: Y^{M} \rightarrow X^{M}$ in the sequence (1.2). This $u$ induces a chain map $u_{M \bullet}: I_{M \bullet} \rightarrow G_{M \bullet}$ with the property that $\mathrm{H}_{-1}\left(u_{M \bullet}\right)=u_{M}$ where $I_{M \bullet}(-1)$ 
and $G_{M} \bullet(-1)$ are the minimal free resolutions of $Y^{M}$ and $X^{M}$ respectively. As for the exact sequence

$$
0 \rightarrow G_{M \bullet} \rightarrow \text { Cone }\left(u_{M \bullet}\right) \bullet(-1) \rightarrow I_{M \bullet}(-1) \rightarrow 0,
$$

we have $\mathrm{H}_{-1}\left(\right.$ Cone $\left.\left(u_{M_{\bullet}}\right)_{\bullet}\right) \cong \operatorname{Ker} u_{M} \cong M, \mathrm{H}_{i}\left(\right.$ Cone $\left.\left(u_{M_{\bullet}}\right)_{\bullet}\right)=0$ for $i \neq-1$ and moreover Cone $\left(u_{M_{\bullet}}\right)_{j}=0$ for $j<-1$. In other words, Cone $\left(u_{M_{\bullet}}\right)_{\bullet}(-1)$ and $F_{M_{\bullet}}$ are quasi-isomorphic. Define the chain map $w_{M_{\bullet}}: G_{M_{\bullet}} \rightarrow F_{M_{\bullet}}$ with the composite $G_{M \bullet} \hookrightarrow$ Cone $\left(u_{M \bullet}\right) \bullet(-1) \rightarrow F_{M \bullet}$. Quite similarly, from the exact sequence

$$
0 \rightarrow F_{M \bullet} \rightarrow \text { Cone }\left(w_{M \bullet}\right) \cdot(-1) \rightarrow G_{M \bullet}(-1) \rightarrow 0,
$$

we have $\mathrm{H}_{-1}\left(\right.$ Cone $\left.\left(w_{M_{\bullet}}\right)_{\bullet}\right) \cong Y^{M}, \mathrm{H}_{i}\left(\right.$ Cone $\left.\left(w_{M_{\bullet}}\right)_{\bullet}\right)=0$ for $i \neq-1$ hence Cone $\left(w_{M_{\bullet}}\right)$, and $I_{M_{\bullet}}$ are quasi-isomorphic. Another chain map $e_{M_{\bullet}}: F_{M_{\bullet}} \rightarrow$ $I_{M \bullet}(-1)$ is defined with the composite $F_{M \bullet} \hookrightarrow$ Cone $\left(w_{M \bullet}\right) \cdot(-1) \rightarrow F_{M \bullet}$. Finally, the exact sequence

$$
0 \rightarrow I_{M \bullet} \rightarrow \text { Cone }\left(e_{M_{\bullet}}\right)_{\bullet}=: \widetilde{G_{M}} \rightarrow F_{M \bullet} \rightarrow 0
$$

gives us $\mathrm{H}_{-1}\left(\right.$ Cone $\left.\left(e_{M_{\bullet}}\right)_{\bullet}\right) \cong X^{M}, \mathrm{H}_{i}\left(\right.$ Cone $\left.\left(e_{M_{\bullet}}\right)_{\bullet}\right)=0$ for $i \neq-1$ and hence Cone $\left(e_{M_{\bullet}}\right)$. and $G_{M \bullet}$ are isomorphic. At this stage, the composite $\operatorname{map} I_{M \bullet} \hookrightarrow \widetilde{G_{M}} \rightarrow G_{M}$. turns back to $u_{M}$ up to homotopy.

As in the proof of Theorem 2.2, we may choose the base of free modules such that

$$
\begin{aligned}
& F_{M i}=W_{M i} \oplus E_{M i}, \quad G_{M i}=U_{M i} \oplus W_{M i}, \quad I_{M i}=E_{M i+1} \oplus U_{M i} \\
& u_{M i}=\begin{array}{c}
U_{M i} \\
W_{M i}
\end{array}\left(\begin{array}{cc}
0 & U_{M i} \\
u^{\prime} & 0
\end{array}\right), \quad u^{\prime} \otimes k=0 . \\
& U_{M i} \quad W_{M i} \\
& w_{M i}={ }_{E_{M i}}^{W_{M i}}\left(\begin{array}{cc}
0 & 1 \\
w^{\prime} & 0
\end{array}\right), \quad w^{\prime} \otimes k=0 . \\
& W_{M i} \quad E_{M i} \\
& e_{M i}=\frac{E_{M i}}{U_{M i-1}}\left(\begin{array}{cc}
0 & 1 \\
e^{\prime} & 0
\end{array}\right), \quad e^{\prime} \otimes k=0 .
\end{aligned}
$$

Note that $E_{M i}=0$ for $i>\operatorname{pd}\left(Y^{M}\right)$ and $U_{M i}=0$ for $i \geq \operatorname{pd}\left(Y^{M}\right)$ thus $w_{M i}=i d_{G_{M i}}$ for $i>\operatorname{pd}\left(Y^{M}\right)$. And the relations among those linear maps are as follows:

$$
d_{F_{M i} i}:=\begin{array}{cc}
W_{M i} & E_{M i} \\
W_{M i-1} & E_{M i-1}
\end{array}\left(\begin{array}{ll}
f_{M 11} & f_{M 12} \\
f_{M 21} & f_{M 22}
\end{array}\right)=\left(\begin{array}{cc}
g_{M 22} & u^{\prime} \\
w^{\prime} e^{\prime} & i_{M 11}
\end{array}\right),
$$




$$
\begin{aligned}
& d_{G_{M i}}:=\begin{array}{cc}
U_{M i} & W_{M i} \\
U_{M i-1} \\
W_{M i-1}
\end{array}\left(\begin{array}{ll}
g_{M 11} & g_{M 12} \\
g_{M 21} & g_{M 22}
\end{array}\right)=\left(\begin{array}{cc}
i_{M 22} & e^{\prime} \\
u^{\prime} w^{\prime} & f_{M 11}
\end{array}\right), \\
& E_{M i+1} \quad U_{M i} \\
& d_{I_{M i}}:=\frac{E_{M i}}{U_{M i-1}}\left(\begin{array}{cc}
i_{M 11} & i_{M 12} \\
i_{M 21} & i_{M 22}
\end{array}\right)=\left(\begin{array}{cc}
f_{M 22} & w^{\prime} \\
e^{\prime} u^{\prime} & g_{M 11}
\end{array}\right)
\end{aligned}
$$

Lemma 3.1 1) The exact sequence (3.26) of complexes induces an exact sequence of modules

$$
0 \rightarrow \Omega_{n+1}^{R}\left(Y^{M}\right) \rightarrow \Omega_{n+1}^{R}\left(X^{M}\right) \oplus E_{M n} \rightarrow \Omega_{R}^{n}(M) \rightarrow 0,
$$

which is the minimal Cohen-Macaulay approximation of $\Omega_{R}^{n}(M)$ for $n \geq$ 0. Thus $\Omega_{R}^{1}\left(X^{M}\right) \stackrel{\text { st }}{\cong} X_{M}$, and $\Omega_{R}^{1}\left(Y^{M}\right) \cong Y_{M}$.

2) The exact sequence (3.25) of complexes induces an exact sequence of modules

$$
0 \rightarrow \Omega_{R}^{n}(M) \rightarrow \Omega_{R}^{n}\left(Y^{M}\right) \oplus \cdot W_{M n-1} \rightarrow \Omega_{R}^{n}\left(X^{M}\right) \rightarrow 0
$$

which is the minimal finite projective hull of $\Omega_{R}^{n}(M)$ for $n \geq 0$. Thus $\Omega_{R}^{n}\left(X^{M}\right) \cong X^{\Omega_{R}^{n}(M)}$ and $\Omega_{R}^{n}\left(Y^{M}\right) \stackrel{s t}{\cong} Y^{\Omega_{R}^{n}(M)}$.

3) The exact sequence (3.24) of complexes induces an exact sequence of modules

$$
0 \rightarrow \Omega_{R}^{n+1}\left(Y^{M}\right) \rightarrow \Omega_{R}^{n}(M) \oplus U_{M_{n-1}} \rightarrow \Omega_{R}^{n}\left(Y^{M}\right) \rightarrow 0,
$$

which is the minimal original extension of $\Omega_{R}^{n}(M)$ for $n \geq 0$ if $\Omega_{R}^{n}(M)$ includes no Cohen-Macaulay module as a direct summand.

proof) The sequence (3.28) (resp. (3.27) ) is obviously a finite projective hull (resp. Cohen-Macaulay approximation), so it remains to show the minimality.

1) minimality of (3.28). The sequence (3.28) is minimal for $n=0$ by definition. If $n>0$, then $\Omega_{R}^{n}\left(X^{M}\right)$ is a stable Cohen-Macaulay module hence cannot contain a common (free), summand with $\Omega_{R}^{n}\left(Y^{M}\right) \oplus W_{M n-1}$.

2)minimality of (3.27). Suppose the contrary; let $E_{M_{n}{ }^{\prime}} \cong R$ be a common summand of $\Omega_{n+1}^{R}\left(X^{M}\right) \oplus E_{M_{n}}$ and $\Omega_{n+1}^{R}\left(Y^{M}\right)$. We may put $E_{M_{n}}=E_{M_{n}}{ }^{\prime} \oplus$ $E_{M n}{ }^{\prime \prime}, \quad I_{M n}=I_{M n}{ }^{\prime} \oplus E_{M n}{ }^{\prime}$ since $\Omega_{n+1}^{R}\left(X^{M}\right)$ is a stable Cohen-Macaulay 
module for $n \geq 0$. Our hypothesis implies that the natural monomorphism

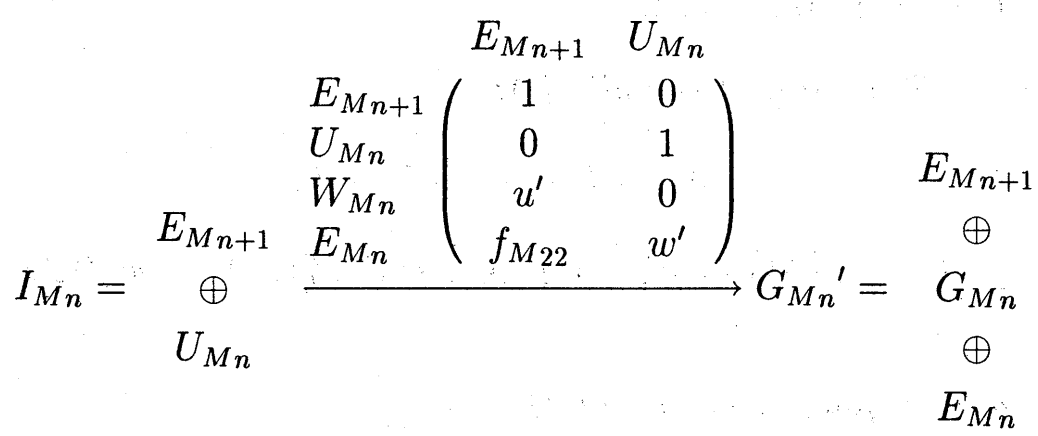

may be isomorphically transformed into

$$
\begin{aligned}
& I_{M n}{ }^{\prime \prime} E_{M n}{ }^{\prime}
\end{aligned}
$$

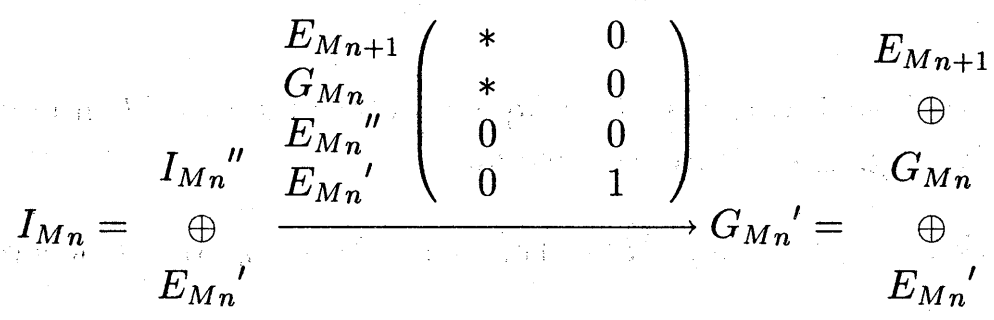

$$
\begin{aligned}
& \begin{array}{c}
\oplus \\
E_{M n} \prime
\end{array}
\end{aligned}
$$

And this base change includes only the row-transformations within the bottom rows corresponding to $E_{M n}$ and column-transformations. Therefore we can transform a matrix $\left(\begin{array}{ll}f_{M_{22}} & w^{\prime}\end{array}\right)$ into $\left(\begin{array}{ll}0 & 0 \\ 0 & 1\end{array}\right)$. It is stupid because $f_{M_{22}} \otimes$ $k=0, \quad w^{\prime} \otimes k=0$. We already have 3) for $n=0$ in Theorem 2.2, and for the higher $n$, it is straightforward since $Y^{\Omega_{R}^{n}(M)} \stackrel{s t}{\cong} \Omega_{R}^{n}\left(Y^{M}\right)$ and $X^{\Omega_{R}^{n}(M)} \cong$ $\Omega_{R}^{n}\left(X^{M}\right)$ from above 1) and 2). (q.e.d.)

With respect to the minimal Cohen-Macaulay approximation (1.1), Auslander defined delta-invariant $\delta_{R}(M)$ as a maximal rank of the free summand in $X_{M}$ and higher delta-invariant $\Omega_{R}^{n}(M):=\delta_{R}\left(\Omega_{R}^{n}(M)\right)$ for $n \geq 0$. From the standpoint regarding a Cohen-Macaulay approximation as one side of triangulated categories, we consider other types of invariants belonging to other two notions.

\section{Definition $3.2 \quad$ 1) For any Cohen-Macaulay approximation of $M$}

$$
0 \rightarrow Y \rightarrow X \rightarrow M \rightarrow 0
$$


put $e_{R}(M):=\mu(Y)-\mu(X)+\mu(M), e_{R}^{i+1}(M):=e_{R}\left(\Omega_{R}^{i}(M)\right)$ for $i \geq 0$, and $e_{R}^{0}(M):=\mu(M)-w(M)$.

2) For any finite projective hull of $M$

$$
0 \rightarrow M \rightarrow Y \rightarrow X \rightarrow 0
$$

Put $w_{R}(M):=\mu(M)-\mu(Y)+\mu(X)$, and $w_{R}^{i}(M):=w_{R}\left(\Omega_{R}^{i}(M)\right)$ for $i \geq 0$.

3) For any original extension of $M$

$$
0 \rightarrow X \rightarrow M \oplus P \rightarrow Y \rightarrow 0
$$

Put $u_{R}(M):=\mu(X)-\mu(M \oplus P)+\mu(Y)$, and $u_{R}^{i}(M):=w\left(\Omega_{R}^{i}(M)\right)$ for $i \geq 0$.

Notice that those invariants are uniquely determined by $M$ independent of the choice of a sequence. Moreover, we have

$$
e_{R}^{i}(M)=\operatorname{rk}\left(e_{M i} \otimes k\right), \quad w_{R}^{i}(M)=\operatorname{rk}\left(w_{M i} \otimes k\right), \quad u_{R}^{i}(M)=\operatorname{rk}\left(u_{M i} \otimes k\right) .
$$

for $i \geq 0$. Remember that $e_{R}^{i}(M)$ is nothing but Auslander's delta-invariants $\delta_{R}^{i}(M)$. In addition, the following are straightforward from the definition.

1)

$$
\begin{gathered}
e_{R}^{i}(M \oplus N)=e_{R}^{i}(M)+e_{R}^{i}(N) \text { for } i \geq 0 \\
e_{R}^{i+j}(M)=e_{R}^{i^{\prime}+j^{\prime}}(M) \text { for } i+j=i^{\prime}+j^{\prime}, i, j, i^{\prime}, j^{\prime} \geq 0 .
\end{gathered}
$$

2)

$$
\begin{gathered}
w_{R}^{i}(M \oplus N)=w_{R}^{i}(M)+w_{R}^{i}(N) \quad \text { for } i \geq 0 \\
w_{R}^{i+j}(M)=w_{R}^{i^{\prime}+j^{\prime}}(M) \quad \text { for } i+j=i^{\prime}+j^{\prime}, i, j, i^{\prime}, j^{\prime} \geq 0
\end{gathered}
$$

3)

$$
\begin{gathered}
u_{R}^{i}(M \oplus N)=u_{R}^{i}(M)+u_{R}^{i}(N) \text { for } i \geq 0 \\
u_{R}^{i+j}(M)=u_{R}^{i^{\prime}+j^{\prime}}(M) \text { for } i+j=i^{\prime}+j^{\prime} ; i, j, i^{\prime}, j^{\prime} \geq 0 .
\end{gathered}
$$

In terms of these invariants, the observation at the beginning of this section is rewritten: 
Remark 3.3

$$
\begin{aligned}
\beta_{R}^{i}(M) & =w_{R}^{i}(M)+e_{R}^{i}(M) \\
\beta_{R}^{i+1}\left(X^{M}\right) & =u_{R}^{i}(M)+w_{R}^{i}(M) \\
\beta_{R}^{i+1}\left(Y^{M}\right) & =e_{R}^{i+1}(M)+u_{R}^{i}(M)
\end{aligned}
$$

where $i \geq 0$ and $\beta_{R}^{i}$ denotes the $i$-th Betti number. Moreover, $\beta^{0}\left(Y^{M}\right)=$ $\beta^{0}\left(X^{M}\right)+e_{R}^{0}(M)$, which is well known. So we put $u_{R}^{-1}:=\beta^{0}\left(X^{M}\right)=\operatorname{rk}\left(u_{M-1} \otimes\right.$ k) for convenience.

Example 3.4 If $M$ is a Cohen-Macaulay module with codimension $r$, that is, $\operatorname{Ext}_{R}^{i}(M, R)=0$ for $i \neq r$, we have

$$
\begin{aligned}
& e_{R}^{i}(M)=e_{R}^{r-i}\left(M^{\vee}\right), \\
& w_{R}^{i}(M)=u_{R}^{r-1-i}\left(M^{\vee}\right) \quad \text { for } 0 \leq i \leq r
\end{aligned}
$$

and

$$
u_{R}^{j}(M)=w_{R}^{r-1-j}\left(M^{\vee}\right) \quad \text { for }-1 \leq j \leq r-1 .
$$

proof)

Let $n: L \rightarrow M$ be a homomorphism of modules, and let $F_{L \bullet} \rightarrow L, F_{M \bullet} \rightarrow$ $M, I_{L \bullet}(-1) \rightarrow Y^{L}, I_{M \bullet}(-1) \rightarrow Y^{M} G_{L \bullet}(-1) \rightarrow X^{L}$ and $G_{M \bullet}(-1) \rightarrow X^{M}$ be the minimal free resolutions. We first take a chain map $n_{F_{\bullet}}: F_{L_{\bullet}} \rightarrow F_{M \bullet}$ with $\mathrm{H}_{0}\left(n_{\bullet}\right)=n$, then two more chain maps $n_{I_{\bullet}}: I_{L_{\bullet}} \rightarrow I_{M \bullet}$ and $n_{G_{\bullet}}: G_{L_{\bullet}} \rightarrow G_{M \bullet}$ induced by the next diagrams.

$$
\begin{array}{cccccc}
L & \rightarrow & M & X_{L} & \rightarrow & X_{M} \\
\downarrow & & \downarrow & \downarrow & & \downarrow \\
Y^{L} & \rightarrow & Y^{M} & L & \rightarrow & M
\end{array}
$$

Since

$$
\mathrm{H}_{i}\left(\text { Cone }(n \bullet)_{\bullet}\right) \cong\left\{\begin{array}{ll}
\text { Coker } n & i=-1 \\
\text { Ker } \mathrm{n} & i=0 \\
0 & i \neq 0,-1
\end{array},\right.
$$

$\tau_{0}$ Cone $\left(n_{\bullet}\right)$ is a free resolution of the module Coker $d_{\text {Cone }}\left(n_{\bullet}\right)_{1}$, whose invariants we can calculate as follows:

Lemma 3.5 Under the situation as above, the following formulae hold.

1)

$$
\begin{aligned}
& e_{R}^{n}\left(\text { Coker } d_{\text {Cone }\left(n_{F}\right)_{1}}\right)=e_{R}^{n+1}(M)+e^{n}(L) \\
& \quad-\operatorname{rk}\left(n_{F n} \otimes k\right)+\operatorname{rk}\left(n_{G n} \otimes k\right)-\operatorname{rk}\left(n_{I n} \otimes k\right)
\end{aligned}
$$


for $n \geq 1$. The left-hand-side of (3.30) is $e_{R}^{n}(\operatorname{Ker} n)$ if $n$ is surjective, while it is $e_{R}^{n+1}(\operatorname{Coker} n)$ if $n$ is injective.

$$
e_{R}^{0}\left(\operatorname{Coker} d_{\text {Cone }\left(n_{F}\right)_{1}}\right)=e_{R}^{1}(M)+e^{0}(L)+\operatorname{rk}\left(n_{G 0} \otimes k\right)-\operatorname{rk}\left(n_{I 0} \otimes k\right) .
$$

The left-hand-side of (3.31) is $e_{R}^{0}\left(\operatorname{Ker} n \oplus F_{M 0}\right)$ if $n$ is surjective. If $n$ is injective, it is $e_{R}^{1}(\operatorname{Coker} n)$ and

$$
e_{R}^{0}(\text { Coker } n)=e_{R}^{0}(M)+\operatorname{rk}\left(n_{G-1} \otimes k\right)-\operatorname{rk}\left(n_{I-1} \otimes k\right) .
$$

2)

$$
\begin{aligned}
& w_{R}^{n}\left(\text { Coker } d_{\text {Cone }\left(n_{F}\right)_{1}}\right)=w_{R}^{n+1}(M)+w_{R}^{n}(L) \\
& \quad-\operatorname{rk}\left(n_{F n+1} \otimes k\right)-\operatorname{rk}\left(n_{G n} \otimes k\right)+\operatorname{rk}\left(n_{I n} \otimes k\right)
\end{aligned}
$$

for $n \geq 0$. The left-hand-side of (3.32) is $w_{R}^{n}(\operatorname{Ker} n)$ if $n$ is surjective, while it is $w_{R}^{n+1}($ Coker $n)$ if $n$ is injective.

3)

$$
\begin{aligned}
& u_{R}^{n}\left(\text { Coker } d_{\text {Cone }\left(n_{F}\right)_{1}}\right)=u_{R}^{n+1}(M)+u_{R}^{n}(L) \\
& \quad+\operatorname{rk}\left(n_{F n+1} \otimes k\right)-\operatorname{rk}\left(n_{G n+1} \otimes k\right)-\operatorname{rk}\left(n_{I n} \otimes k\right)
\end{aligned}
$$

for $n \geq 0$. The left-hand-side of (3.33) is $u_{R}^{n}(\operatorname{Ker} n)$ if $n$ is surjective, while it is $u_{R}^{n+1}($ Coker $n)$ if $n$ is injective.

proof) As for the chain maps $w_{L_{\bullet}}: G_{L_{\bullet}} \rightarrow F_{L_{\bullet}}$ and $w_{M_{\bullet}}: G_{M_{\bullet}} \rightarrow F_{M_{\bullet}}$, the following diagram commutes up to homotopy:

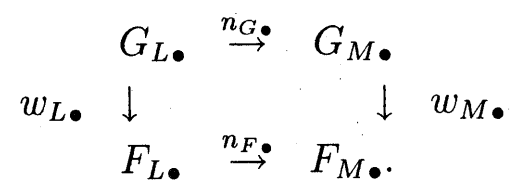

And we get a commutative diagram with exact rows and columns :

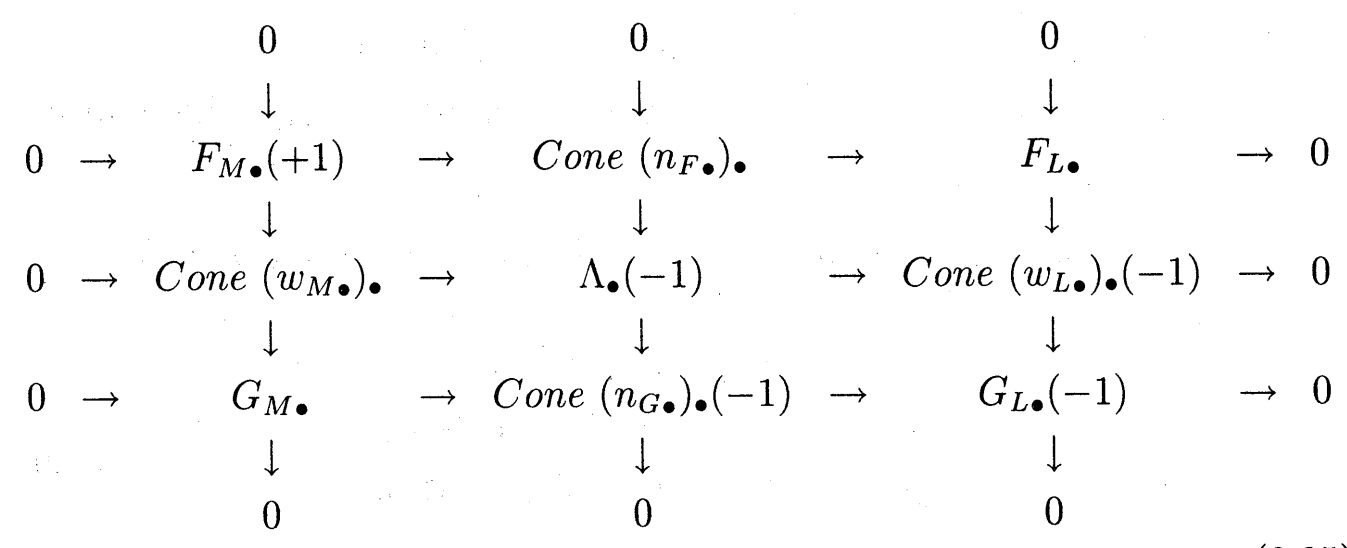


The complex $\Lambda_{\bullet}$ is obtained as a mapping cone;

$$
\Lambda_{\bullet}:=\text { Cone }\left(n_{\tilde{I}_{\bullet}}\left(h_{w}\right)\right)_{\bullet} \cong \text { Cone }\left(\hat{w}_{\bullet}\left(h_{W}\right)\right) \bullet
$$

where $n_{\tilde{I}_{\bullet}}\left(h_{w}\right)$ : Cone $\left(w_{L_{\bullet}}\right)_{\bullet} \rightarrow$ Cone $\left(w_{M_{\bullet}}\right)_{\bullet}$ and $\hat{w}_{\bullet}\left(h_{w}\right):$ Cone $\left(n_{G_{\bullet}}\right)_{\bullet} \rightarrow$ Cone $\left(n_{F_{\bullet}}\right)$ are defined as follows;

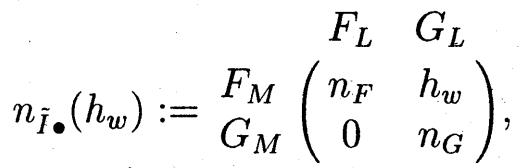

$$
\begin{aligned}
& G_{M} \quad G_{L} \\
& \hat{w}_{\bullet}\left(h_{w}\right):=\begin{array}{l}
F_{M} \\
F_{L}^{\prime}
\end{array}\left(\begin{array}{cc}
w_{M} & h_{w} \\
0 & w_{L}
\end{array}\right),
\end{aligned}
$$

using a chain homotopy $h_{w \bullet}$;

$$
n_{F_{\bullet}} w_{L \bullet}-w_{M \bullet} n_{G \bullet}=i_{\bullet} d_{G_{L}}+d_{F_{M}} i_{\bullet}
$$

These chain maps are determined uniquely up to homotopy, independent of the choice of $h_{W}$; for another homotopy $h_{w}{ }^{\prime}$, since $h_{w}-h_{w}{ }^{\prime}: G_{L \bullet} \rightarrow F_{M \bullet}(+1)$ is a chain map, the universal property of Cohen-Macaulay approximation gives a chain map $j_{\bullet}: G_{L_{\bullet}} \rightarrow G_{M_{\bullet}}(+1)$ and a chain homotopy $h_{\bullet}: G_{L_{\bullet}} \rightarrow F_{M_{\bullet}}(+2)$ such that $h_{w \bullet}-h_{w}{ }^{\prime} \bullet=w_{M \bullet} j_{\bullet}+h_{\bullet} d_{G_{L}}+d_{F_{M} \bullet}$, which induces the equation

$$
\left(\begin{array}{cc}
0 & h_{w}-h_{w}^{\prime} \\
0 & 0
\end{array}\right)=\left(\begin{array}{cc}
0 & -h \\
0 & -j
\end{array}\right)\left(\begin{array}{cc}
d_{F_{L}} & w_{L} \\
0 & -d_{G_{L}}
\end{array}\right)+\left(\begin{array}{cc}
d_{F_{M}} & w_{M} \\
0 & -d_{G_{M}}
\end{array}\right)\left(\begin{array}{ll}
0 & h \\
0 & j
\end{array}\right) .
$$

From the middle column of (3.35 we get a finite projective hull of Coker $d_{\text {Cone }}\left(n_{F}\right) \bullet n+1$;

$$
0 \rightarrow \operatorname{Coker} d_{\text {Cone }\left(n_{F}\right)_{\bullet n+1}} \rightarrow \operatorname{Coker} d_{\Lambda_{n}} \rightarrow \operatorname{Coker} d_{\text {Cone }\left(n_{G_{\bullet}}\right)_{n}} \rightarrow 0
$$

since other two columns also induce finite projective hulls as we see in Lemma 3.1. We have only to look at the number of generators to calculate

$$
\begin{aligned}
& w_{R}^{0}\left(\operatorname{Coker} d_{\text {Cone }}\left(n_{F_{\bullet}}\right)_{n+1}\right) \\
& =\mu\left(\operatorname{Coker} d_{\text {Cone }\left(n_{F_{\bullet}}\right)_{n+1}}\right)-\mu\left(\operatorname{Coker} d_{\Lambda n}\right)+\mu\left(\operatorname{Coker} d_{\text {Cone }\left(n_{G}\right)_{n}}\right)
\end{aligned}
$$

In the matrix form,

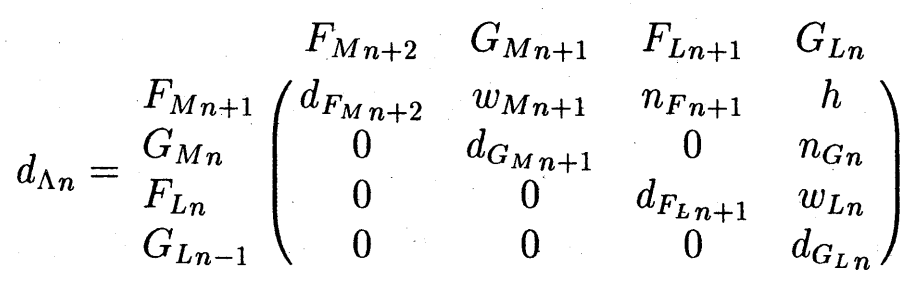


can be rewritten as

$$
\begin{aligned}
& W_{M n+2} \quad I_{M_{n+1}} \quad W_{M n+1} \quad W_{L n+1} \quad I_{L n} \quad W_{L n} \\
& \left.d_{\Lambda n}=\begin{array}{l}
W_{M n+1} \\
I_{M n} \\
W_{M n} \\
W_{L n}
\end{array} \quad \begin{array}{ccccccc}
0 & 0 & 1 & p_{11} & p_{12} & p_{13} \\
& 0 & d_{I_{M n+1}} & 0 & p_{21} & p_{22} & p_{23} \\
& 0 & 0 & 0 & p_{31} & p_{32} & p_{33} \\
W_{L n-1} & 0 & 0 & 0 & 0 & 0 & 1 \\
0 & 0 & 0 & 0 & d_{I_{L n}} & 0 \\
0 & 0 & 0 & 0 & 0 & 0
\end{array}\right)
\end{aligned}
$$

after the base changes of Cone $\left(w_{M}\right)$. and Cone $\left(w_{L}\right)_{n-1}$. The right upper part corresponds to the chain map $\tilde{n}:$ Cone $\left(w_{M}\right)_{\bullet} \rightarrow$ Cone $\left(w_{L}\right)_{n-1}$ hence satisfying

$$
\tilde{n} d_{\text {Cone }\left(w_{L}\right)}=d_{\text {Cone }\left(w_{L}\right)} \tilde{n},
$$

that is,

$$
\left(\begin{array}{ccc}
0 & p_{12 n} d_{I_{L}} & p_{11_{n}} \\
0 & p_{2 n_{n}} d_{I_{L}} & p_{21_{n}} \\
0 & p_{32 n} d_{I_{L}} & p_{31 n}
\end{array}\right)=\left(\begin{array}{ccc}
p_{31} & p_{32} & p_{33} \\
d_{I_{M}} p_{21} & d_{I_{M}} p_{22} & d_{I_{M}} p_{23} \\
0 & 0 & 0
\end{array}\right)
$$

The above equation shows that

$$
p_{21} \otimes k=0, \quad p_{32} \otimes k=0, \quad p_{31}=0,
$$

so we have

$$
\operatorname{rk}\left(d_{P n} \otimes k\right)=\operatorname{rk}\left(W_{M n+1} \oplus W_{L n}\right)+\operatorname{rk}\left(p_{22_{n}} \otimes k\right) .
$$

On the other hand, $p_{22}: I_{L} \rightarrow I_{M}$ is a chain map and coincides with $n_{I_{\bullet}}$ up to homotopy in view of the following commutative diagram.

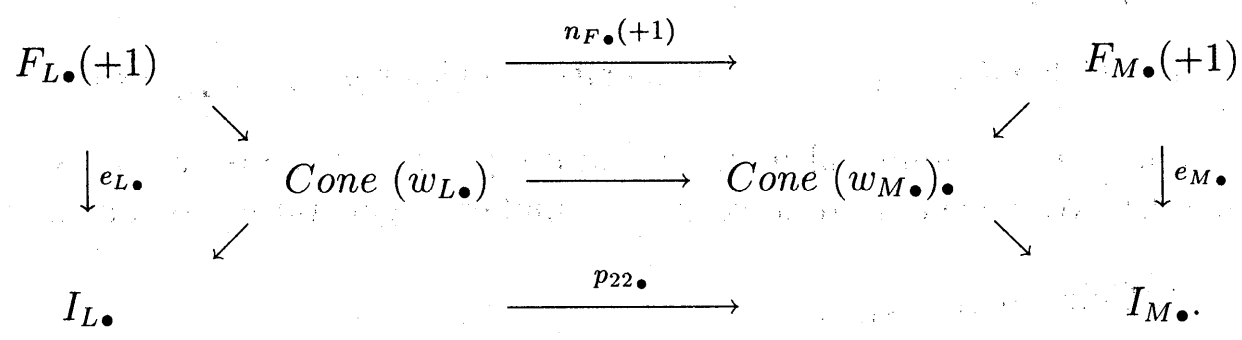

Hence the above equation is

$$
\operatorname{rk}\left(d_{P n} \otimes k\right)=w_{R}^{n+1}(M)+w_{R}^{n}(L)+\operatorname{rk}\left(n_{I_{n}} \otimes k\right) .
$$

Together with $\mu\left(\right.$ Coker $\left.d_{\text {Cone }\left(n_{F}\right)_{n}}\right)=\operatorname{rk}\left(\right.$ Cone $\left.\left(n_{F}\right)_{n}\right)-\operatorname{rk}\left(n_{F n+1} \otimes k\right)$ and $\mu\left(\right.$ Coker $\left.d_{\text {Cone }\left(n_{G}\right)_{n}}\right)=\operatorname{rk}\left(\right.$ Cone $\left.\left(n_{G}\right)_{n-1}\right)-\operatorname{rk}\left(n_{G n} \otimes k\right)$, we have

$$
\begin{aligned}
& w_{R}^{0}\left(\text { Coker } d_{\text {Cone }}\left(n_{F_{\bullet}}\right)_{n+1}=w_{R}^{n}\left(\text { Coker } d_{\text {Cone }}\left(n_{F} \bullet\right)_{1}\right.\right. \\
& =w_{R}^{n+1}(M)+w_{R}^{n}(L)-\operatorname{rk}\left(n_{F n+1} \otimes k\right)-\operatorname{rk}\left(n_{G n} \otimes k\right)+\operatorname{rk}\left(n_{I n} \otimes k\right)
\end{aligned}
$$


and also

$$
\begin{aligned}
& e_{R}^{0}\left(\text { Coker } d_{\text {Cone }\left(n_{F}\right)_{n+1}}\right)=\mu\left(\text { Coker } d_{\Lambda n}\right)-\mu\left(\operatorname{Coker} d_{\text {Cone }\left(n_{G}\right)_{n}}\right) \\
& =e_{R}^{n+1}(M)+e_{R}^{n}(L)+\operatorname{rk}\left(n_{G n} \otimes k\right)-\operatorname{rk}\left(n_{I n} \otimes k\right)
\end{aligned}
$$

as required. Parallel discussions give the proofs for other invariants. (q.e.d.)

We use this method especially on the lifting problem. Let $R:=S / x S$ with a Gorenstein local ring $S$ and a non-zero-divisor $x$. For an $R$-module $M$, the relation between invariants of $M$ as an $R$-module and those as $S$-module is described via Eisenbud operators $\partial_{F_{M}}$, $\partial_{I_{M}}$, and $\partial_{G_{M}}$. with respect to $S, x$.

\section{Corollary 3.6}

$$
\begin{aligned}
& e_{S}^{n}(M)=e_{R}^{n}(M)+e_{R}^{n-1}(M)-\operatorname{rk}\left(\partial_{F_{M n}} \otimes k\right)+\operatorname{rk}\left(\partial_{G_{M n}} \otimes k\right)-\operatorname{rk}\left(\partial_{I_{M n}} \otimes k\right) . \\
& w_{S}^{n}(M)=w_{R}^{n}(M)+w_{R}^{n-1}(M)-\operatorname{rk}\left(\partial_{F_{M n+1}} \otimes k\right)-\operatorname{rk}\left(\partial_{G_{M n}} \otimes k\right)+\operatorname{rk}\left(\partial_{I_{M n}} \otimes k\right) . \\
& u_{S}^{n}(M)=u_{R}^{n}(M)+u_{R}^{n-1}(M)+\operatorname{rk}\left(\partial_{F_{M n+1}} \otimes k\right)-\operatorname{rk}\left(\partial_{G_{M n+1}} \otimes k\right)-\operatorname{rk}\left(\partial_{I_{M n}} \otimes k\right) .
\end{aligned}
$$

Lemma 3.7 ([4] Lemma 3.1) The following isomorphisms holds for $n \geq 0$ :

$$
\Omega_{R}^{n+1}\left(Y_{R}^{M}\right) \cong \Omega_{R}^{n}\left(Y_{M}^{R}\right) \cong Y_{\Omega_{R}^{n}(M)}^{R} .
$$

proof) We show that $\Omega_{R}^{1}\left(Y_{R}^{M}\right) \cong Y_{M}^{R}$. The minimal Cohen-Macaulay approximation (1.1) gives us the push out diagram as below:

$$
\begin{aligned}
& \begin{array}{rr}
0 & 0 \\
\downarrow & \downarrow \\
Y_{M} & =\quad Y_{M}
\end{array} \\
& \gamma_{M} \downarrow \quad \gamma^{\prime} \downarrow
\end{aligned}
$$

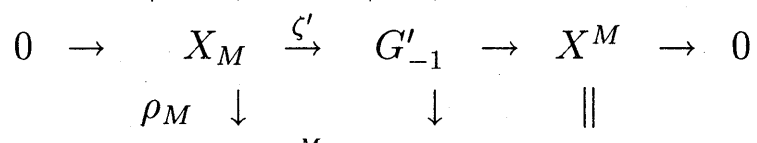

$$
\begin{aligned}
& \begin{array}{rrr}
M & \stackrel{\zeta^{M}}{\rightarrow} & Y^{M} \\
\downarrow & \downarrow & \\
\downarrow & & \\
0 & & 0
\end{array}
\end{aligned}
$$

Here $G_{-1}$ is an $R$-free module and we may take the cosyzygy as $\zeta^{\prime} \otimes k=0$, $X^{M}$ is a stable Cohen-Macaulay module. Then $\gamma^{\prime} \otimes k=0$. If otherwise, there exists a homomorphism $s: G_{-1}^{\prime} \rightarrow Y_{M}$ such that $s \gamma^{\prime} s=s$. Applying $\gamma_{M}$, we have $\gamma_{M} s \gamma^{\prime} s=\left(\gamma_{M} s\right) \zeta^{\prime}\left(\gamma_{M} s\right)=\left(\gamma_{M} s\right)$ which contradicts to $\zeta^{\prime} \otimes k=0$.

Similarly we can prove $\Omega_{R}^{1}\left(Y_{M}^{R}\right) \cong Y_{\Omega_{R}^{1}(M)}^{R}$ and the induction on $n$ completes the proof. (q.e.d.) 


\section{References}

[1] M.Auslander and R.O.Buchweitz, The homological theory of maximal Cohen-Macaulay approximations, Soc. Math. de France, Mem 38(1989), $5-37$.

[2] M.Auslander, S.Ding, and Ø.Solberg, Liftings and weak liftings of modules, J.Algebra 156 (1993), 273-317.

[3] D.Eisenbud, Homological algebra on a complete intersection with an application to group algebra, Trans. Amer. Math Soc. 260 (1980), 35-64.

[4] K.Kato, Vanishing of free summands in Cohen-Macaulay approximations, to appear in Comm.Algebra.

[5] Y.Yoshino, "Cohen-Macaulay modules over Cohen-Macaulay rings," London Math.Soc., Lecture Note Series vol.146, Cambridge U.P., 1990.

[6] Y.Yoshino, The theory of L-complexes and weak liftings of complexes, preprint, 1994. 